DOE/ER/40145--7

DE93 001798

\title{
RESEARCH IN THEORETICAL NUCLEAR PHYSICS
}

\begin{tabular}{|c|c|c|}
\hline $\begin{array}{c}\text { Takeshi Udagawa } \\
\text { Theoretical Nuclear Physics Group } \\
\text { Department of Physics } \\
\text { University of Texas } \\
\text { Austin, Texas, } 78712\end{array}$ & 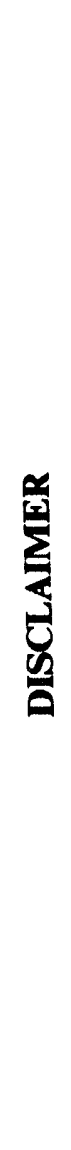 & 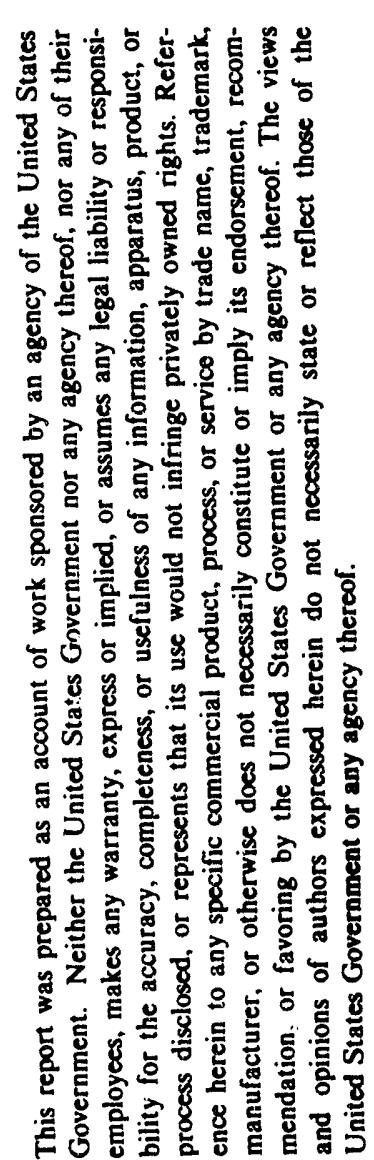 \\
\hline
\end{tabular}

PREPARED FOR THE U.S. DEPARTMENT OF ENERGY

UNDER GRANT NUMBER DE-FG05-84ER40145 


\section{TABLE OF CONTENTS}

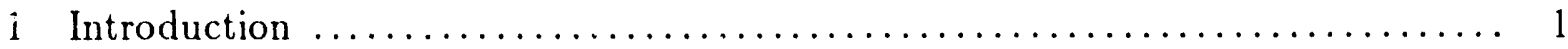

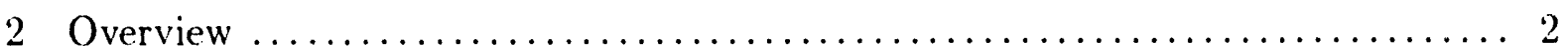

2.1 Low Energy Nuclear Reactions Induced by Light and Heavy lons ..... 2

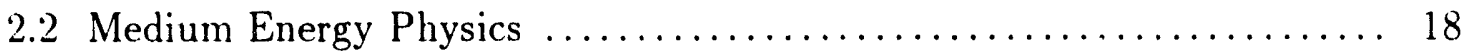

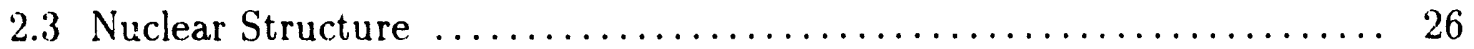

3 Title Pages of Published Papers $\ldots \ldots \ldots \ldots \ldots \ldots \ldots \ldots \ldots \ldots \ldots \ldots \ldots . \ldots \ldots$

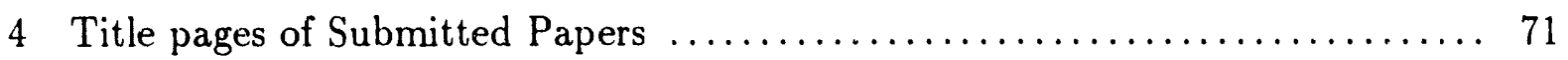

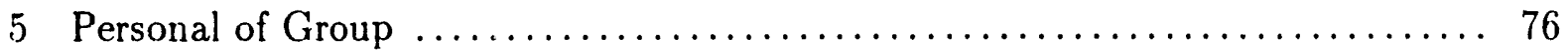




\section{INTRODUCTION}

This report describes the accomplishments in basic research in nuclear physics carried out by the theoretical nuclear physics group in the Department of Physics at The University of Texas at Austin, during the period of November 1, 1989 to September 30, 1992 under grant DE-FG05-84ER40145 between the University of Texas at Austin and the United States Department of Energy.

The major part of this report is contained within sections 2,3 , and 4 . Section 2 is a presentation of our research achievements and their significance. Both work completed and in progress are covered. In section 3 , title pages of papers published during the period are reproduced, while in section 4, first pages of papers submitted but not yet in print are reproduced. These papers are numbered as 3-1 through 3-26 and 4-1 through 4-4, according to the order which they appear in these two sections.

Section 2 gives an overview of our work, its interrelations, and its relation to work done by other groups. As seen, a variety of subjects are included in our work, and thus the description is presented in three separate parts; (2.1) through (2.3). Each part includes brief introductory remarks, followed by the description of work done on specific topics. The main topic in each part can be found in the list of contents given at the beginning of this report. In the presentations in section 2, frequent references are made to papers in sections 3 and 4 , and also to many other papers. 


\section{OVERVIEW}

\subsection{Low Energy Nuclear Reactions Induced by Light and Heavy Ions}

One of the most important goals of nuclear reaction theories is to establish an approach for analyzing experimental data from different types of nuclear reactions, i.e., complete fusion (CF), incomplete fusion (IF) and direct reactions (DR), in a systematic way based on a single theoretical framework. In an attempt to achieve this goal, we have developed a few techniques for calculating cross sections for $\mathrm{CF}$ and various types of IF reactions within the framework of DR theory. As is well known, the DR theory itself can be formulated on the basis of the unified theory of nuclear reactions. Within this theory, the fusion part of the reaction process is incorporated in the imaginary part of the optical potential (though such a description can account for the energy averaged behavior only). This is the key ingredient in our approach.

The technique developed for the IF reaction calculation is the breakup-fusion (BF) approach. In this approach, we assume that the reaction proceeds as breakup of the incident particle $a$ into, say, $b+x$, followed by absorption of $x$ by the target $A, b$ being the emitted particie. In other words, the IF reaction is viewed as a partial fusion (or more precisely, reaction) taking place in three (or more) body channels that are reached by breakup (or by some other DR, knockout, for instance). We then treat this partial fusion using the imaginary part of the optical potential $W_{x}$ for the $x+A$ system. Since the first breakup reaction is itself a typical DR, it can be treated by the usual DR theory. It is thus possible to calculate the whole BF process within the framework of the DR theory.

The technique developed for calculating the fusion cross section, which is called the DR approach to fusion (DRAF), also utilizes the imaginary part of the optical potential. More specifically, fusion is treated as absorption by the inner part of the imaginary potential $W$. We call this ininer portion of $W$ the fusion potential $W_{F}$. The remainder (outer part) of $W$ may then be ascribed to the IF-plus-DR processes, which we call the DR part. We then calculate the total fusion and DR cross sections, $\sigma_{F}$ and $\sigma_{D}$ respectively, as $\sigma_{i}=(2 / \hbar v)\left\langle\chi^{(+)}\left|W_{i}\right| \chi^{(+)}\right\rangle$ $(i=\mathrm{F}$ or $\mathrm{D})$, where $\chi^{(+)}$is the optical model wave function.

The major part of the work described in the following subsections concerns the application, testing, and improvement of these theoretical methods. 
2.1.1 Calculations of Complete Fusion, Incomplete Fusion and Direct Reaction Cross Sections within the Framework of Direct Reaction Theories

Using the two techniques discussed in the introductory remarks, i.e., the BF approach ${ }^{1}$ and $\mathrm{DRAF}^{2}$, together with the multistep direct reaction (MSDR) method, ${ }^{3}$ developed also by our group, it is possible to calculate the total non-compound nuclear reaction cross section $\sigma_{D}$ by two different methods. One approach is to use DRAF, while the other is to calculate cross sections of all possible IF and DR reactions using the BF and DR methods, and then sum the individual cross sections. Let us write the $\sigma_{D}$ 's obtained by these two methods as $\sigma_{D}^{D R A F}$ and $\sigma_{D}^{I F+D R}$, respectively. An important issue here is whether these two cross sections mutually agree or not. Furthermore, we may question whether the regions from whence the dominant contributions to these cross sections originate are the same in the two approaches.

In collaboration with Dr.Mastroleo at Instituto de Estudos Avancados, Sao Jose dos Campos, Brazil and Dr.Mustafa at Lawrence Livermore National Laboratory, we recently tried to answer the above question, ${ }^{4}$ taking as an example the $\mathrm{d}+{ }^{93} \mathrm{Nb}$ system. Fig. 1 shows the results of the calculated $\sigma_{D}^{D R A F}$ and $\sigma_{D}^{I F+D R}$ for $E_{d}=10 \sim 25 \mathrm{MeV}$. Use was made of a fusion potential radius of $R_{F}=7.2 \mathrm{fm}$ (or $r_{F}=1.58 \mathrm{fm}$ ) in obtaining $\sigma_{D}^{D R A F}$. With this choice for the $R_{F}$-value, good agreement between the two theoretical predictions was obtained. We then examined the radial regions where the elastic breakup (EB) and $\mathrm{BF}$ reactions dominantly occur. The results showed that the centers of the radial region where the $\mathrm{EB}$ and $\mathrm{BF}$ reactions take place are $10 \mathrm{fm}$ and $8.8 \mathrm{fm}$ respectively. The resulting centers are well outside the fusion potential radius $R_{F}=7.2 \mathrm{fm}$, used for the DRAF calculations. Thus both approaches reach a consistent conclusion about the regions where the IF and DR predominantly occur in the incident channel. This shows that the two approaches are mutually consistent.

1. T. Udagawa and T. Tamura, Phys. Rev. C 24, 1348 (1981).

T. Udagawa and T. Tamura, Phys. Rev. C 33, 494 (1986).

T. Udagawa, X.-H. Li and T. Tamura, Phys. Rev. C 37, 429 (1988).

2. T. Udagawa, B. T. Kim and T. Tamura, Phys. Rev. C 32, 124 (1984).

3. T. Tamura, T.Udagawa, and H. Lenske, Phys. Rev. C 26, 379 (1982). 
4. R. C. Mastroleo, T. Udagawa, and M. Mustafa, Phys. Rev. C 42, 683 (1990). (See also 3-1.) R. C. Mastroleo, T. Udagawa, and M. Mustafa, Proc. of Intern. Symp. on Nuclear Collective Motion and Nuclear Reaction Dynamics, held at Riken, Japan, eús. by K.-I. Kubo, M. Ishihara, M. Ichimura, and S. Yamaji (World Scientific, 1991), p.270. (See also 3-2.)

\subsubsection{Dynamical Calculations of Linear Momentum Transfer in $\alpha$-Induced Reactions}

One remarkable result of the calculations reported in the previous subsection is that the sum of the calculated total incomplete fusion (IF) and direct reaction (DR) cross sections, $\sigma_{I F}$ and $\sigma_{D R}$, respectively, amounts to about $50 \%$ of the total reaction cross section, $\sigma_{R}$. Since $\sigma_{I F}+\sigma_{D R}$ well approximates the total non-compound nuclear cross section, we reach the conclusion that the complete fusion (CF) cross section, $\sigma_{C F}$, evaluated as $\sigma_{C F}=\sigma_{R}-$ $\sigma_{I F}-\sigma_{D R}$ is about $50 \%$ of $\sigma_{R}$. This is a sizable reduction of $\sigma_{C F}$ from $\sigma_{R}$. Consider that $\sigma_{C F}$ is a crucial ingredient in the usual statistical model calculation of emission cross sections of various particles from the decay of the compound nucleus, ${ }^{1}$ where $\sigma_{R}$ is customarily used in place of $\sigma_{C F}$. It is expected then that if use is properly made of $\sigma_{C F}$, the calculated emission cross sections will significantly change. In this respect, it is important to test the validity of the above result for $\sigma_{C F}$.

Unfortunately, there are presently no experimental data available for $\sigma_{C F}$. Therefore, it is not possible to make a direct test of the calculated $\sigma_{C F}$ against the data. It is, however, possible to make an indirect test via comparison of the calculated linear momentum transfer per nucleon, $p / a$, with experiment, since $p / a$ is very sensitive to $\sigma_{C F}$. Note that we can calculate $p / a$ in a consistent manner by using the $\sigma_{C F}$ calculated as $\sigma_{C F}=\sigma_{R}-\sigma_{I F}-\sigma_{D R}$ and also various DR and IF cross sections evaluated in the course of obtaining the total $\sigma_{I F}$ and $\sigma_{D R}$.

Such a test was indeed recently attempted, ${ }^{2}$ taking the $\alpha+{ }^{59} \mathrm{Co}$ system ${ }^{3}$ with an incident energy $E_{a}=80 \mathrm{MeV}$, as an example. The calculated $p / a$-value was found to be $163 \mathrm{MeV} / \mathrm{c}$, which agreed well with the experimental value of $160 \mathrm{MeV} / \mathrm{c}$. We note that the dominant contribution $(69 \%)$ of the calculated $p / a$ comes from CF. The good agreement of the calculated $p / a$ with the experimental data thus strongly supports the validity of the calculated 
$\sigma_{C F}\left(=967 \mathrm{mb}\right.$, which is $58 \%$ of $\left.\sigma_{R}\right)$. We also note that the various DR and IF cross sections used in obtaining the above $p / a$-value have also been tested against the data taken for the $\alpha+{ }^{58} \mathrm{Ni}$ system. ${ }^{4}$

Using the calculated value of $\sigma_{C H}=967 \mathrm{mb}$, one can go one step further to estimate the radius, $R_{F}$, of the fusion potential, $W_{F}$, introduced in our direct reaction approach to fusion (DRAF). ${ }^{5}$ (See also the discussion given in in the introductory remark and previous subsection.) $R_{F}$ can be determined by fitting the calculated $\sigma_{C F}$ within DRAF to the above $\sigma_{C F}=967 \mathrm{mb}$. The $R_{F}$-value thus deduced is $R_{F}=6.57 \mathrm{fm}$, which corresponds to a reduced radius parameter of $r_{F}=1.69 \mathrm{fm}$. The $r_{F}$-value thus obtained is not very far from the value of $1.58 \mathrm{fm}$ determined previously for the $\mathrm{d}+{ }^{93} \mathrm{Nb}$ system with $E_{d}=10-25 \mathrm{MeV},{ }^{6}$ as discussed in the previous subsection 2.1.1. Note that these two values are both larger than the typical nuclear radius parameter, say, $r_{0}=1.2 \mathrm{fm}$, indicating that fusion begins at a fairly large distance. This conclusion is in very good agreement with what was concluded for heavy-ion fusion. ${ }^{6}$

As already argued above, the fact that $\sigma_{C F}$ is significantly smaller than $\sigma_{R}$ has an important influence on the calculated cross sections of particles emitted from the highly excited compound nucleus. This is most likely the case since we along with other research groups have demonstrated that some of the difficulties encountered can be removed by the use of $\sigma_{C F}$ or equivalently the transmission coefficient evaluated from DRAF. ${ }^{1,5}$ The work described in this and previous subsections, together with that discussed in the subsequent sections, was summarized $^{7}$ recently at the International Conference held in honour of the Late Professor Taro Tamura, at RIKEN, Japan, December 18-20, 1989.

1. N. G. Nicolis et. al., Phys. Rev. C 45, 2393 (1992).

2. R. C. Mastroleo, T. Udagawa, and M. G. Mustafa, Phys. Lett. B 245, 329 (1990). (See also 3-3.)

3. J. Jastrzebski, P. P. Singh, T. Mróz, S. E. Vigdor, M. Fatyga, and H. J. Karwowski, Phys. Rev. C 34, 60 (1986).

4. J. R. Wu et al., Phys. Rev. C 20, 1284 (1979).

5. T. Udagawa, B. T. Kim, and T. Tamura, Phys. Rev. C 32, 124 (1981).

6. M. G. Mustafa, T. Tamura, and T. Udagawa, Phys. Rev. C 35, 2067 (1989). 
7. T. Udagawa, Proc. of Intern. Symp. on Nuclear Collective Motion and Nuclear Reaction Dynamics, held at Riken, Japan, eds. by K.-I. Kubo, M. Ishihara, M. Ichimura, and S. Yamaji (World Scientific, 1991), p.181. (See also 3-4.)

\subsubsection{Breakup-Fusion Analyses of the ${ }^{40} \mathrm{Ca}\left({ }^{6} \mathrm{Li}, \mathrm{d}\right){ }^{44} \mathrm{Ti}$ Reactions and $\alpha$-Cluster Structure in ${ }^{44} \mathrm{Ti}$}

The breakup-fusion (BF) approach employed in the studies reported in the previous subsections was originally developed for the description of the incomplete-fusion reaction, i.e., a stripping-type transfer reaction to the unbound region. The approach was, however, shown ${ }^{1,2}$ to be applicable to calculate cross section for stripping reactions to the bound region also. In fact, the approach provides a unified description of stripping reactions to both bound and unbound regions. The method was then applied to analyze single nucleon transfer reaction data, demonstrating that it is even capable of extracting spectroscopic information for high-lying single particle states.

Recently, we have extended the analysis ${ }^{3,4}$ to data of the $\alpha$-transfer ${ }^{40} \mathrm{Ca}\left({ }^{6} \mathrm{Li}, \mathrm{d}\right){ }^{44} \mathrm{Ti}$ reactions at incident energies of 28,50 , and $60 \mathrm{MeV} .^{4-6}$ The aim is to extract spectroscopic information, particularly information about the $\alpha$-cluster structure in ${ }^{44} \mathrm{Ti}$, which is of current interest. ${ }^{7-12}$ Since many of the $\alpha$-cluster states appear in the unbound region, use of the $\mathrm{BF}$ method is most appropriate for the purpose. From the analysis of the data, transfered angular momenta $\ell_{\alpha, i}$ and spectroscopic factors $S_{i}$ have been extracted for the individual final states up to $E_{\text {ex }} \approx 10 \mathrm{MeV}$.

The relative $S$-factors deduced from the data with the three different incident energies agreed well with each other. The most remarkable result is an identification of a $0^{+}$state at $E_{e x}=9.32 \mathrm{MeV}$, (identified only in the analysis of the $60 \mathrm{MeV}$ data). We have assigned the state to be the $0^{+}$member of the $\mathrm{N}=14$ band. From this assignment we were further able to estimate the absolute magnitude of the $S$-factors for the lowest $0^{+}-12^{+}$states of the $N=12$ band. The values are $S=0.45,0.38,0.19,0.30,0.21,0.19$ and 0.32 , respectively, which agree fairly well with the theoretical values of $S=0.36,0.41,0.40,0.29,0.35,0.31$ and 0.23 obtained by Arima and Tomoda. ${ }^{13}$ We were also able to confirm for the first time in the 
$\left({ }^{6} \mathrm{Li}, \mathrm{d}\right)$ reaction that the $8.04 \mathrm{MeV}$ state is the $12^{+}$member of the $\mathrm{N}=12$ band. The $S$-factors have been obtained for a fairly large number of $J^{\pi}=0^{+}, 2^{+}, 4^{+}$and $3^{-}$states. The results, together with observation of the $\mathrm{N}=140^{+}$state at $E_{e x}=9.32 \mathrm{MeV}$, strongly suggesi that the cluster strength of $\mathrm{N}=12$ and 13 is widely spread into many states.

The conclusions drawn in the study described above are still not sufficiently solid. In order to test them further, additional studies are needed, particularly in the determination of the absolute magnitude of the $S$-factors. One possible way to accomplish this is to extend the analysis to the continuum region. There it is expected that the $\alpha$-cluster picture of the ${ }^{6} \mathrm{Li}$ $\rightarrow \alpha+d$ process becomes more valid, and therefore we may obtain more reliable information on the overall normalization constant from the analysis of such data. Unfortunately, such data are not available at present. The extension of the present measurement to highly excited continuum states is thus highly desirable.

1. T. Udagawa, Y. J. Lee, and T. Tamura, Phys. Lett. B 196, 291 (1987).

2. T. Udagawa, Y. J. Lee, and T. Tamura, Phys. Rev. C 39, 1 (1989).

3. Chong-Yeal Kim and T. Udagawa, Phys. Rev. C 46, 532 (1992) (See also 3-5.)

4. P. Guazzoni, M. Jaskola, L. Zetta, Chong-Yeal Kim, T. Udagawa, and G. Bohlen, to be published. (See also 4-1.)

5. H. W. Fulbright, C. L. Bennet, R. A. Lindgren, R. G. Markham, S. C. McGuire, G. C. Morrison, U. Strohbusch, and J. Töke, Nucl. Phys. A284, 329 (1977).

6. T. Yamaya, S. Oh-ami, M. Fujiwara, T. Itahashi, K. Katori, M. Tosaki, S. Saito, S. Hatori, and S. Ohkubo, Phys. Rev. C 42, 1935 (1990).

7. F. Michel, G. Reidemeister, and S. Ohkubo, Phys. Rev. Lett. 57, 1215 (1986).

8. F. Michel, G. Reidemeister, and S. Ohkubo, Phys. Rev. C 38, 2377 (1988).

9. A. C. Merchant, K. F. Pál, and P. E. Hodgson, J. Phys. G. 15, 605 (1989).

10. A. Arima, Proc. Topical Conference on Physics of Medium Light Nuclei, ed. by P. Blasi and R. Ricci (Editrice Compositori, Bologna, 1979) p.19.

11. T. Wada, and H. Horiuchi, Phys. Rev. C 38, 2063 (1988).

12. S. Ohkubo, Proc. of 6th International Conference on Nuclear Reaction Mechanisms, ed. by E. Gadioli (Universita Degli Studio Di Milano, 1991) p.396.

13. T. Tomoda and A. Arima, Nucl. Phys. A303, 217 (1978). 


\subsubsection{Breakup of ${ }^{7} \mathrm{Li}$ into $\alpha$-t Continuum at Relative Energy of $0 \sim 2 \mathrm{MeV}$}

This is a continued effort in collaboration with the experimental group at Texas A\&M University. The subject is the breakup of ${ }^{7} \mathrm{Li}$ into $\alpha$ and $t$, which has been studied by several authors ${ }^{1,2}$, because of the interest in the value of the so-called astrophysical $S$-factor for very small relative energies $\varepsilon(=10 \sim 200 \mathrm{keV})$ in the $\alpha$-t continuum. In principle, the $S$-factor could be determined by measuring the cross section of the direct $\gamma$-capture process $\alpha(\mathrm{t}, \gamma)^{7} \mathrm{Li}^{3,4}$ Since the cross section is extremely small at small $\varepsilon$, however, it has been difficult to measure the cross section in this way.

Baur, et al., proposed to use Coulomb breakup data to determine the $S$-factor indirectly. ${ }^{2}$ Using a newly developed technique, Utsunomiya, et al..$^{5-7}$, at Texas A\&M performed measurements of the Coulomb breakup of ${ }^{7} \mathrm{Li}$. There 42 and $63 \mathrm{MeV}$ beams of ${ }^{7} \mathrm{Li}$ were bombarded on various targets such as ${ }^{208} \mathrm{~Pb},{ }^{144} \mathrm{Sm},{ }^{120} \mathrm{Sn},{ }^{58} \mathrm{Ni}$, and ${ }^{27} \mathrm{Al}$. The breakup cross section were determined as functions of the relative energy $\varepsilon$ and the angle of the $\alpha-t$ system.

We have analyzed the data for the yields to the continuum at $\varepsilon=0 \sim 1.0 \mathrm{MeV}$, attempting to extract the astrophysical $S$-factors as a function of $\varepsilon$. In the most recent analysis, ${ }^{7}$ we introduced an idea that the nuclear-Coulomb interference effects do not significantly change the $\varepsilon$-dependence of the Coulomb excitation cross section. We tested the validity of this idea by making a DWBA calculation, confirming that it is indeed valid. The energy dependence of the resultant $S$-factors then agreed very well with those deduced from the $(\gamma, \mathrm{p})$ reaction data of Ref. 4.

1. A. C. Shotter et al., Phys. Rev. Lett. 53, 1539 (1981)

2. G. Baur, C. A. Bertulani and H. Rebel, Nucl. Phys. A458, 188 (1986).

3. G. M. Griffiths et al., Can. J. Phys. 39, 1397 (1961).

4. U. Schroder et al., Phys. Lett. B192, 55 (1987).

5. H. Utsunomiya, R. P. Schmitt, Y.-W. Lui, D. R. Haenni, H. Dejbakhsh, L. Cooke, P. Heimberg, A. Ray, T. Udagawa and T. Tamura, Phys. Lett. B211, 24 (1988).

6. H. Utsunomiya, Y.-W. Lui, L. Cooke, H. Dejbakhsh, D. R. Haenni, P. Heimberg, A. Ray, B. K. Srivastava, R. P. Schmitt, and T. Udagawa, Nucl. Phys. A511, 379 (1990). (See also 3-6.)

7. H. Utsunomiya, Y.-W. Lui, D. R. Haenni, H. Dejbakhsh, L. Cooke, B. K. Srivastava, W. Turmel, D. O'Kelly, R. P. Schmitt, D. Shapira, J. Gomez del Campo, A. Ray, and T. Udagawa, Phys. Rev. Lett. 65, 847 (1990). (See also 3-7.) 


\subsubsection{Dispersive Optical Model Potentials for Subbarrier Heavy Ion Fusion and Scattering}

Analyses of the subbarrier fusion cross section $\sigma_{F}$ based on the direct reaction approach to fusion (DRAF) ${ }^{1}$ have been one of the major subjects of our heavy ion reaction studies during the past several years. Very recently, we have initiated a new type of analysis, i.e., the simultaneous $\chi^{2}$-analysis ${ }^{2-4}$ of elastic scattering $\left(\sigma_{E L}\right)$, fusion $\left(\sigma_{F}\right)$, and total direct reaction (DR) cross section $\left(\sigma_{D}\right)$ data. The optical potential used in such an analysis consists of a volume-type real potential, $V_{R}(r)$, a volume-type imaginary fusion potential, $W_{F}(r)$, and a surface-derivative-type imaginary $\mathrm{DR}$ potential, $W_{D}(r)$. The parameters of these potentials are then fixed from the $\chi^{2}$-analysis of $\sigma_{E L}, \sigma_{F}$, and $\sigma_{D}$. In DRAF, $\sigma_{i}(\mathrm{i}=\mathrm{D}$ and F) is calculated within the optical model as $\sigma_{i}=(2 / \hbar v)\left\langle\chi^{(+)}\left|W_{i}(r)\right| \chi^{(+)}\right\rangle$, where $v$ is the incident velocity and $\chi^{(+)}$is the elastic distorted wave function.

Such analyses have so far only been made for the two systems, ${ }^{16} \mathrm{O}+{ }^{208} \mathrm{~Pb}$ and ${ }^{32} \mathrm{~S}+{ }^{58,64} \mathrm{Ni}$. The results obtained are, however, very encouraging, revealing many interesting features in $V_{R}, W_{F}$ and $W_{D}$, which in turn provide valuable information on the dynamics of the nuclear reactions involved. For instance, the radius, $r_{F}$, of the fusion potential $W_{F}$ turned out to be quite large $\left(r_{F}=1.40 \mathrm{fm}\right)$, larger than the typical radius $(1.30 \mathrm{fm})$ for the Coulomb-barrier top, indicating that fusion starts to occur at a large distance. Extracted values for $W_{F}$ at the strong absorption radius $R_{A}, W_{F}\left(R_{A}\right)$, showed very clearly the so-called threshold anomaly ${ }^{5}$, i.e., $W_{F}\left(R_{A}\right)$ showed a sudden decrease when the incident energy $E$ went below the Coulomb barriei height. Further, $W_{D}$ showed a very interesting system-dependence, reflecting the dynamical features of the systems considered. Moreover, the real and imaginary potentials satisfied very well the required dispersion relation ${ }^{6,7}$ at $r=R_{A}$. (Very recently, iv came to our attention that some of the older data used in the analyses of Ref. 3 do not agree with new data taken very recently at different laboratories. ${ }^{8,9}$ This means that we need to repeat our analysis. We believe, however, that the qualitative features of $W_{D}$ and $W_{F}$ determined in Ref. 3 will remain unchanged in the new $W_{D}$ and $W_{F}$.)

Recently, we went one step further to obtain a fully dispersive optical potential, i.e., an optical potential that satisfies the dispersion relation over all space. ${ }^{4}$ This was done for the ${ }^{16} \mathrm{O}+{ }^{208} \mathrm{~Pb}$ system. The optical potential used consisted of the Coulomb potential, $U_{C}(r)$, 
the energy-independent bare (Hartree-Fock or folding) potential, $V_{0}(r)$, and the real and imaginary polarization potentials, $V(r)$ and $W(r)$, respectively, that describe the coupledchannel (CC) effects. The polarization potentials are further assumed to have volume-type fusion and surface-derivative-type DR parts; $V(r)=V_{F}(r)+V_{D}(r)$ and $W(r)=W_{F}(r)+$ $W_{D}(r)$.

The optical potential assumed above involves eleven parameters, excluding the Coulomb radius parameter in $U_{C}$, which we chose as $r_{c}=1.25 \mathrm{fm}$. It was impossible to determine all eleven parameters uniquely from a $\chi^{2}$-analysis. We thus fixed a priori some of the parameters; the parameters of the bare potential $V_{0}(r)$ were fixed as $V_{0}=60.4 \mathrm{MeV}, r_{0}=1.176 \mathrm{fm}$, and $a_{0}=0.658 \mathrm{fm}$, while all the geometrical parameters in the polarization potential $V(r)+i W(r)$ were fixed at $r_{F}=1.40 \mathrm{fm}, a_{F}=0.25 \mathrm{fm}, r_{D}=1.50 \mathrm{fm}$, and $a_{D}=0.45 \mathrm{fm}$. These values are chosen as the best possible values, taking into consideration the results of earlier studies. ${ }^{3,10}$ Note, however, that these values are still not unique, and this non-uniqueness might introduce a certain ambiguity in the analyses made in Ref. 4.

Since the geometrical parameters in $V_{i}(r)$ and $W_{i}(r)(i=F$ and $D)$ are assumed to be the same, the dispersion relations hold for the strength parameters $V_{i}$ and $W_{i}$. The relation reads 6,7

$$
V_{i}(E)=V_{i}\left(E_{s}\right)+\frac{E-E_{s}}{\pi} F \int_{0}^{\infty} d E^{\prime} \frac{W_{i}\left(E^{\prime}\right)}{\left(E^{\prime}-E_{s}\right)\left(E^{\prime}-E\right)}
$$

where $\mathrm{P}$ stands for the principal value and $V_{i}\left(E_{s}\right)$ is the value of the potential at a reference energy $E=E_{s}$. Using the above relation, it is possible to evaluate values of $V_{F}$ and $V_{D}$, once the values of $W_{F}(E)$ and $W_{D}(E)$ are known. This means that we now have only two parameters to be fixed from the analysis.

Fig. 2 shows values of $W_{F}(E)$ and $W_{D}(E)$ determined from the $\chi^{2}$-fit. These $W_{F}(E)$ and $W_{D}(E)$ values can be well represented by the following functions of $E$ (in units of $\mathrm{MeV}$ ):

$$
W_{F}(E)= \begin{cases}0 & \text { for } E \leq 77.5 \\ 0.52(E-77.5) & \text { for } 77.5<E \leq 84 \\ 3.4 & \text { for } 84 \leq E\end{cases}
$$




$$
W_{D}(E)= \begin{cases}0 & \text { for } \mathrm{E} \leq 70 \\ 0.185(E-70) & \text { for } 70<\mathrm{E} \leq 90 \\ 0.3 i & \text { for } 90 \leq \mathrm{E}\end{cases}
$$

The solid lines shown in Fig. 2 are the $W_{F}$ and $W_{D}$ values predicted by the above Eqs. (2) and (3). As seen, the lines fit the empirical values (shown by the filled circles) rather well. In obtaining $V_{F}$ and $V_{D}$ from Eq. (1) use was thus made of the above functional forms for $W_{F}(E)$ and $W_{D}(E)$, together with $V_{F}\left(E_{s}\right)=1.8 \mathrm{MeV}$ at $E_{s}=84 \mathrm{MeV}$ and $V_{D}\left(E_{s}\right)=0.5 \mathrm{MeV}$ at $E_{s}=90 \mathrm{MeV}$.

In Fig. 2, it is seen that the slope (0.185) of $W_{D}$ is much smaller than that of $W_{F}(0.52)$. This means that the E-dependence of $W_{D}$ is much weaker than that of $W_{F}$. This clearly reflects the observed fact that the energy-dependence of the experimental $\sigma_{D}$ is much weaker than that of $\sigma_{F}$. This, together with the fact that the $W_{F}$ dominates the total imaginary potential, $W\left(=W_{F}+W_{D}\right)$, at least in the higher energy part of the Coulomb-barrier energy region, shows that the rapid energy change in $W$ originates from fusion. Namely, the main cause of the threshold anomaly comes from the coupling of the elastic channel with fusion channels.

In Figs. 3 and 4 , we present the final fit of the calculated $R=\sigma_{E L} / \sigma_{R U T H}$ (where $\sigma_{R U T H}$ is the Rutherford cross section), $\sigma_{F}$ and $\sigma_{D}$ to the experimental data. As seen, the fit is good. The dotted line shown for one case in Fig. 3 is the $R$-value obtained by setting $V_{D}=0$. As seen, when $V_{D}$, which is one of the new elements in the analyses, is set to zero, the fit of the calculated $R$ to experiment becomes worse, showing that $V_{D}$ plays an important role. Note that the deviation of the dotted line from the full line is seen only in the angle region between, say, $\theta_{1}$ and $\theta_{2}$, where $\theta_{1}$ is the angle at which $R$ starts to decrease while $\theta_{2}$ is the angle where the value of $R$ becomes $R \sim 10^{-2}$. This region corresponds to the radial region, where $V_{D}$ has an appreciable value.

The work described above was presented ${ }^{11}$ at the international workshop on subbarrier fusion held at Daresbury, June 5-7, 1990. 
1. T. Udagawa, B. T. Kim and T. Tamura, Phys. Rev. C 32, 124 (1984).

2. S. W. Hong, T. Udagawa and T. Tamura, Nucl. Phys. A491, 492 (1989).

3. T. Udagawa, T. Tamura and B. T. Kim, Phys. Rev. C 39, 1840 (1989).

4. B. T. Kim, M. Naito, and T. Udagawa, Phys. Lett. B 237, 19 (1990). (See also 3-8.)

5. J. S. Lilley, B. R. Fulton, M. A. Nagarajan, I. J. Thompson, and D. W. Banes, Phys. Lett. B 151181 (1985).

6. C. Mahaux, H. Ngo and G. R. Satchler, Nucl. Phys. A449 (1986) 354; Nucl. Phys. A456, 134 (1986).

7. M. A. Nagarajan, C. Mahaux and G. R. Satchler, Phys. Rev. Lett. 54, 1136 (1985).

8. R. J. Tighe et al., Phys. Rev. C 42, 1530 (1990)

9. Y. Sugiyama, private communication.

10. G. R. Satchler, M. A. Nagarajan, J. S. Lilley and I. J. Thompson, Ann. of Phys. 178, 110 (1987).

11. T. Udagawa and B. T. Kim, Inst. Phys. Conf. Ser. No 110, (IOP Publishing Ltd, 1991), 103. (See also 3-9.)

\subsubsection{Systematics of Sub-Barrier Fusion Cross Sections and the Threshold Anomaly}

One of the consequences found in the study reported in the previous subsection 2.1 .5 is that the main cause of the threshold anomaly ${ }^{1}$ is fusion, i.e., the coupling of the elastic channel to fusion channels. We have found additional evidence of this, which was reported recently. ${ }^{2}$ The evidence is that the threshold energy $E_{F}$ of the subbarrier fusion cross section, $\sigma_{F}$, agrees very well with the threshold energy $E_{0}$ of the imaginary potential $W$. The threshold anomaly is of course seen in $W$, which is extracted from an analysis of elastic scattering data. As noted previously, $W$ at the strong absorption radius $R_{A}$ can be very well represented in the sub-barrier region by a linear function of $E$ as $W\left(R_{A}\right)=\alpha\left(E-E_{0}\right)$. The zero intercept $E_{0}$ then defines the threshold energy of $W$. Stelson et al. ${ }^{3}$ observed the quantity $S$ defined as $S=\left(E \sigma_{F}\right)^{1 / 2}$ can also be very well represented by a linear function of $E$ as $S=\beta\left(E-E_{F}\right)$ in the sub-barrier region. $E_{F}$ then defines the threshold energy $E_{F}$ of the sub-barrier fusion.

We have tried ${ }^{1}$ to collect information on the $\left(E_{F}, E_{0}\right)$-value. We have so far been able to obtained such values for three systems, ${ }^{16} \mathrm{O}+{ }^{60} \mathrm{Ni},{ }^{16} \mathrm{O}+{ }^{63} \mathrm{Cu}$ and ${ }^{16} \mathrm{O}+{ }^{208} \mathrm{~Pb}$. The values obtained are: $\left(E_{F}, E_{0}\right)=(29.2,29.3),(30.2,31.0)$ and $(71.3,69.0) \mathrm{MeV}$, respectively, for the above three system. As seen, the $E_{F^{-}}$and $E_{0^{-}}$-values agree well with each other. 
$E_{F}$ is a quantity characterizing the threshold of the fusion reaction. The above agreement of $E_{0}$ with $E_{F}$ thus indicates that the strong energy dependence seen in $W\left(E, R_{A}\right)$ in the threshold energy region (threshold anomaly) indeed comes from fusion.

1. J. S. Lilley, B. R. Fulton, M. A. Nagarajan, I. J. Thompson, and D. W. Banes, Phys. Lett. B 151, 181 (1985).

B. R. Fulton, D. W. Banes, J. S. Lilley, M. A. Nagarajan, I. J. Thompson, Phys. Lett. B 162, 55 (1985).

2. T. Udagawa, M. Naito, and B. T. Kim, Phys. Rev. C 45, 876 (1992). (See also 3-10.)

3. P. H. Stelson, H. J. Kim, M. Beckerman, D. Shapira, and R. L. Robinson, Phys. Rev. C 41, 1584 (1990).

P. H. Stelson, Phys. Lett. B 205, 190 (1988).

\subsubsection{Dynamical Fusion and Direct Reaction Potentials For Heavy-Ion Scattering and Fusion at Sub-Coulomb-Barrier Energies}

In subsection 2.1.5, we have reported results of our simultaneous $\chi^{2}$-analyses ${ }^{1-3}$ of elastic scattering, fusion and total direct reaction (DR) cross section data taken at sub- and nearCoulomb-barrier energies. One important result obtained from the analyses is that the fusion potential $W_{F}(r)$, assumed to have a Woods-Saxon shape should have a large radius parameter $r_{F} \approx 1.4 \mathrm{fm}$, placing the resulting radius $R_{F}$ beyond the peak in the Coulomb barrier. The value is much larger than that usually assumed in the barrier penetration model ${ }^{4}$ or coupledchannels (CC) calculations ${ }^{5,6}$ for fusion. Satchler, et al., ${ }^{7}$ then proposed that the large $r_{F}$ values have their origin in the known importance of couplings to DR channels. In a CC approach, the so-called "bare" imaginary fusion potential $W_{O F}(r)$ may still be confined inside the Coulomb barrier and have a small radius, $r_{0 . r}=1.0 \mathrm{fm}$. If one projects, however, this CC problem onto the elastic channel, there appears an additional term, $\Delta W_{F}$, in the resultant fusion potential. This is a part of the dynamical polarization potential (DPP), $\Delta U$, which appears when such a projection is done. The proposal made by Satchler, et al., ${ }^{7}$ is that $r_{F}$ becomes large because of $\Delta W_{F}$ in $\Delta U$.

A study ${ }^{8}$ was recently made to test whether $\Delta W_{F}(r)$ indeed makes the $r_{F}$-value of the resultant fusion potential $\left(W_{0 F}+\Delta W_{F}\right)$ much larger than $r_{0 F}$. For this purpose, we calculated 
$\Delta U$, particularly the fusion and DR potentials, $\Delta W_{F}$ and $\Delta W_{D}$, respectively, where $\Delta W_{D}$ is the DR part in the total imaginary potential in $\Delta U$. The actual numerical calculations were carried out for the ${ }^{16} \mathrm{O}+{ }^{208} \mathrm{~Pb}$ system at $E_{\text {lab }}=80 \mathrm{MeV}$, taking into account couplings with the $2.61 \mathrm{MeV} \mathrm{3}^{-}, 3.20 \mathrm{MeV}^{-}$, and $4.07 \mathrm{MeV}^{+}$states in ${ }^{208} \mathrm{~Pb}$, and to tise $6.13 \mathrm{MeV} 3^{-}$ state in ${ }^{16} \mathrm{O}$ using the collective form factor.

The resultant $\Delta W_{F}$ and $\Delta W_{D}$ are found to be highly nonlocal and $\ell$-dependent. Because of this, it was difficult to reach a conclusion on Satchler's hypothesis. To see the area where fusion and DR take place more clearly, we thus examined the following absorption densities $A_{i}(r)(i=F$ and $D)$,

$$
A_{i}(r)=\int_{0}^{\infty} \Delta W_{i}\left(\ell ; r, r^{\prime}\right) R e\left[\rho_{\ell}\left(r, r^{\prime}\right)\right] d r^{\prime} . \quad\left(\rho_{\ell}\left(r, r^{\prime}\right) \equiv \chi_{\ell}^{*}(r) \chi_{\ell}\left(r^{\prime}\right)\right)
$$

The above expression can be derived from the divergence of the current density. Figs. 5a and $5 \mathrm{~b}$ show the calculated $A_{F}(r)$ and $A_{D}(r)$ as functions of $r$ for the $\ell=10$ case. Tle DR absorption density $A_{D}(r)$ is appreciable in the region of $r>12 \mathrm{fm}$. It has an oscillatory long range tail. The period of oscillation reflects that of the distorted wave function function $\chi_{\ell}(r)$, and the long range tail comes from Coulomb excitation. The nuclear excitation contribution is confined in the peak region of $12 \mathrm{fm}<r<14 \mathrm{fm}$. The center of the peak is located at $r \approx 13 \mathrm{fm}$. This region is simply the grazing region where DR are supposed to occur.

The fusion absorption density $A_{F}(r)$ also has an oscillatory structure. The period of oscillation reflects that of the nonlocal density $R e\left[\rho\left(r, r^{\prime}\right)\right]$ in Eq. (1). The envelope of oscillation, peaked at $r \approx 9.7 \mathrm{fm}$, is roughly proportional to $v_{\ell \ell^{\prime}}^{2}(r) W_{0 F}(r)\left|\chi_{\ell}(r)\right|^{2}$, where $v_{\ell \ell^{\prime}}^{2}(r)$ is the channel coupling potential, the limit of $A_{F}(r)$ obtained when the Green's function is assumed to be a $\delta$-function (adiabatic assumption). The region where $A_{F}(r)$ takes an appreciable value is about $8 \mathrm{fm}<r<12 \mathrm{fm}$, where $v_{\ell \ell^{\prime}}(r)$ and $W_{O F}(r)$ strongly overlap. (Note that the peak of $v_{\ell \ell^{\prime}}(r)$ appears at $R_{0}=9.8 \mathrm{fm}$ of $V_{0}(r)$, while $W_{0 F}(r)$ has $R_{0 F}=8.4 \mathrm{fm}$.) Particularly, the region where $A_{F}(r)$ takes a large positive value (absorption region) is limited to $r \leq 10.2 \mathrm{fm}$. This absorptive region is somewhat shifted outward as compared with the "bare" fusion region defined by $W_{0 F}\left|\chi_{\ell}\right|^{2}$, but still stays inside the barrier (the top of the Coulomb barrier $\approx 11.4 \mathrm{fm}$ ). It is much inside the fusion region predicted in our model ${ }^{3}$ with $r_{F}=1.4 \mathrm{fm}\left(R_{F}=11.8 \mathrm{fm}\right)$. Note that, next to this absorptive region, there appears a 
creative region $(10<r<12 \mathrm{fm})$ where $A_{F}(r)$ becomes negative. The existence of such a creative region may effectively reduce the overall effects from the channel-couplings on fusion. Therefore, the result does not support the idea proposed in Ref. 7.

In Ref. 8, we have also studied in some details the question whether the calculated $\ell$ dependent, non-local potentials can be localized in a reasonable manner. The answer was negative; it is improbable that such a highly nonlocal DPP, like the one obtained in the study of Ref. 8 , can be localized in a meaningful manner. ${ }^{9}$ As remarked, the DPP is also $\ell$-dependent. In Ref. 9 a very elaborate effort was made to obtain localized and $\ell$-averaged DPP's. Unfortunately, the potentials obtained are unable to reproduce consistently the calculated $\mathrm{CC}$ elastic scattering cross sections.

In contrast to this, phenomenologically we have always been successful in finding a local, $\ell$-independ $\epsilon$ t optical potential that can reproduce the experimental elastic scattering, fusion and total DR cross section data. Apparently, it has not been possible so far to derive such a local, $\ell$-independent phenomenological potentia: directly from $\mathrm{CC}$ calculations. One possible origin of this failure may be ascribed to the insufficient number of channels included in the $\mathrm{CC}$ calculations and the use of a too simplified form of the coupling scheme (neglecting the coupling tetween inelastic channels, etc.). One might generally expect ${ }^{10}$ that if one includes more channels and uses a more realistic coupling scheme, the complicated nonlocality may be washed away. The Green's functions in the different inelastic channels have different oscillations in $r$-space and the resulting potential becomes more local because of the randomphase nature of the contributions from a large number of terms. The $\ell$-dependence may also be averaged out. It would be interesting and important if such calculations could be done. The work described here was presented at the international conference on nuclear dynamics held at Nikko, Japan. ${ }^{11}$ 
1. T. Udagawa, B. T. Kim and T. Tamura, Phys. Rev. C 32, 124 (1985). (See also 3-8.)

2. T. Udagawa, T. Tamura and B. T. Kim, Phys. Rev. C 39, 1840 (1989).

3. B. T. Kim, M. Naito, and T. Udagawa, Phys. Lett. B237, 19 (1990).

4. J. R. Birkelund and J. R. Huizenga, Ann. Rev. Nucl. Part. Sci. 33, 265 (1983).

5. S. G. Steadman and M. J. Rhodes-Brown, Ann. Rev. Nucl. Part. Sci. 36, 649 (1986).

S. Landowne and S. C. Pieper, Phys. Rev. C 29, 1352 (1984).

6. G. R. Satchler, et al., Ann. Phys. 178, 110 (1987)

7. G. R. Satchler, et al., Phys. Rev. C 41, 1869 (1990).

8. B. T. Kim and T. Udagawa, Phys. Lett. B 273, 37 (1991). (See also 3-11.)

9. I. J. Thompson, et al., Nucl. Phys. A505, 84 (1989).

10. L. Car.ton, Y. Hahn, and G. Cattapan, Phys. Rev. C 43, 2441 (1991).

11. B. T. Kim and T. Udagawa, Proc. of Inter. Conf. on Towards a Unified Picture of Nuclear Dynamics, ed. by Abe et al., (AIP, New York 1991), p.133. (See also 3-12.)

\subsubsection{A Rapid Method for Nonlocal Optical Model Calculations}

Theoretical nuclear optical potentials are known to be nonlocal in general. ${ }^{1}$ The ronlocality arises from the exchange of particles between the projectile and target (knockon exchange) and from coupling to non-elastic channels. The contribution from the latter channel-coupling (CC) is often referred to as a dynamic polarization potential, and is given by the familiar Feshbach formula. ${ }^{1}$

The nonlocality of the polarization potential $U_{p}$ often becomes very large. ${ }^{2-7}$ An example is provided for the ${ }^{16} \mathrm{O}+{ }^{208} \mathrm{~Pb}$ system at sub- and near-Coulomb barrier energies ${ }^{8}$ as discussed in the previous subsection. As remarked there, the nonlocality of the potential calculated in Ref. 8 is so large that it is difficult to localize it in a reasonable manner. In such cases, it is necessary to do a nonlocal calculation.

In a recent article ${ }^{9}$ we proposed a new, rapid, and reliable method for solving the nonlocal optical model Schrödinger equation: $\left(E-H_{\ell}\right)\left|\chi_{\ell}\right\rangle=U^{\prime}\left|\chi_{\ell}\right\rangle$, where $\chi_{\ell}$ is the partial wave function that we wish to evaluate, $H_{\ell}$ is the partial wave Hamiltonian, which may include a local optical potential $U_{0}$, and $U^{\prime} \equiv U-U_{0}, U$ being the nonlocal potential. The basic idea of the approach is to modify the above Schrödinger equation into the foll swing integral equation for $\left|\lambda_{\ell}\right\rangle$ defined as $\left|\lambda_{\ell}\right\rangle=U^{\prime}\left|\chi_{\ell}\right\rangle$;

$$
\left|\lambda_{\ell}\right\rangle=\left|\rho_{\ell}\right\rangle+U^{\prime} G_{\ell}\left|\lambda_{\ell}\right\rangle \quad\left(\left|\rho_{\ell}\right\rangle \equiv U^{\prime}\left|\chi_{\ell}^{(0)}\right\rangle\right)
$$


where $G_{\ell}=\left(E-H_{\ell}+i \epsilon\right)^{-1}$, while $\left|\chi_{\ell}^{(0)}\right\rangle$ is the solution of the local optical model Schrödinger equation defined by $H_{\ell}$.

We solve the above Eq. (1) using the Lanczos method, ${ }^{11}$ in which $\left|\lambda_{\ell}\right\rangle$ is expanded in terms of an orthonormal set of basic vectors $\left|D_{i}\right\rangle$. We generate such basic vectors iteratively starting with the doorway state vector $|\rho\rangle ;\left|D_{0}\right\rangle=\left(1 / N_{0}\right)|\rho\rangle$, where $N_{0}$ is a normalization constant. The validity of this approach depends on the number $N$ of the basic vectors needed in the expansion in order to obtain enough accuracy. We examined this point for two example cases; one being the nonlocal problem considered by Perey and Buck ${ }^{12}$ and the other was the problem encountered when a coupled-channels problem is reduced to a one dimensional problem. For the later problem, we took the ${ }^{16} \mathrm{O}+{ }^{208} \mathrm{~Pb}$ system at the incident energy of $E_{l a b}=80 \mathrm{MeV}$, and considered the simple case of coupling to the $3^{-}$state in ${ }^{208} \mathrm{~Pb}$ at an excitation energy of $2.6 \mathrm{MeV}$. The first example is a rather simple nonlocal problem, while the second case is a highly nonlocal problem. The $\mathrm{N}$-values required for obtaining a good accuracy $(0.1 \%)$ for the first and second example cases were 3 and 8 for, respectively. The $\mathrm{N}$-value for the second is larger than that for the first example, which is due to the complex nature of the nonlocality of the potential considered in the later case. Nevertheless, the Nvalues are rather small for both cases, indicating the method is valid for both. A computer program has been developed for numerical calculations. ${ }^{13}$

1. H. Feshbach, Ann. of Phys. (N. Y.) 5357 (1958), ibid, 19, 287 (1962).

2. C. L. Rao, M. Reeves, and G. R. Satchler, Nucl. Phys. A207, 182 (1973).

3. P. W. Coulter and G. R. Satchler, Nucl. Phys. A293, 269 (1977).

4. N. Vinh Mau and A. Bouyssy, Nucl. Phys. A257, 189 (1976).

5. F. Osterfeld, J. Wambach, and V. A. Madsen, Phys. Rev. C 23, 179 (1981).

6. R. S Mackintosh and S.G. Cooper, Nucl. Phys. A494, 123 (1989).

7. I. J. Thompson, M. A. Nagarajan, J. S. Lilley, and M. J. Smithson, Nucl. Phys. A505, 84 (1989).

8. B. T. Kim and T. Udagawa, Phys. Lett. B 273, 37 (1991) (See also 3-11.)

9. B. T. Kim and T. Udagawa, Phys. Rev. C 42, 1147 (1990). (See also 3-13.)

10. T. Udagawa and B. T. Kim, Phys. Rev. C 40, 2271 (1989).

11. R. R. Whitehead, A. Watt, B. J. Cole, and I. Morrison, Adv. Nucl. Phys. 9, 123 (1977).

12. F. Perey and B. Buck, Nucl. Phys. 32, 353 (1962).

13. B. T. Kim, M. C. Kyum, S. W. Hong, M. H. Park, and T. Udagawa, to be published in Com. Phys. Comm. (See also 4-2.) 


\subsection{Medium Energy Physics}

The work reported here was done in collaboration with Osterfeld at KFA in Jülich, Germany and Hong at Sung Kyung Kwan University, Seoul. The work concerns nuclear medium effects on $\Delta$ excited in nuclei by means of the $(\mathrm{p}, \mathrm{n})-,(\mathrm{d}, 2 \mathrm{p})$ - and $\left({ }^{3} \mathrm{He}, \mathrm{t}\right)$-charge-exchange reactions and electron scattering at intermediate energies. We shall report on our calculations of the inclusive and exclusive cross sections, and spin observables taking into account the nuclear mean field and two-body delta-hole interactions.

\subsubsection{Inclusive Spectra of $\Delta$-Excitations in Nuclei}

The shift of the $\Delta$ peak position observed in inclusive spectra of the charge-exchange $(\mathrm{p}, \mathrm{n})^{1},\left({ }^{3} \mathrm{He}, \mathrm{t}\right)^{2}$, and $(\mathrm{d}, 2 \mathrm{p})^{3}$ reactions for nuclear targets crimpared to the proton target has been one of the most important issues in medium energy physics over the last several years. The $\Delta$ peak in the proton target spectrum appears at $\omega_{L} \approx 370 \mathrm{MeV}$, where $\omega_{L}$ is the energy transfer in the laboratory system. On the other hand, for a nuclear target $\left({ }^{12} \mathrm{C}\right)$ the peak appears at $\omega_{L} \approx 300 \mathrm{MeV}$. The peak position of $\omega_{L} \approx 370 \mathrm{MeV}$ for the proton target can be explained by the sum of the $\Delta$ resonance energy $\omega_{R}=294 \mathrm{MeV}$, the $\Delta$ recoil energy of $95 \mathrm{MeV}$ received by the $\Delta$ in the $(\mathrm{p}, \mathrm{n})$ excitation process, and an additional shift $(-19 \mathrm{MeV})$ due to momentum-transfer-dependent effects in the $t_{N N, N \Delta}$ transition operator. The downward energy shift of about $70 \mathrm{MeV}$ in nuclear targets has been a mystery to be solved.

Clearly the shift observed is due to nuclear medium effects, and recently we have made ${ }^{4}$ a fairly realistic calculation of the energy spectra of the charge exchange reactions taking into account nuclear mean field and two-body type delta-hole interaction effects. The resui $\iota^{\circ}$ has indicated that about $40 \mathrm{NieV}$ out of the $70 \mathrm{MeV}$ shift are due to Fermi motion (binding) of the target nucleons and the attractive one-body potential for a $\Delta$ (the nucleus mean field effect). A nuclear correlation effect produced by the $\Delta$-particle-nucleon-hole $\left(\Delta N^{-1}\right)$ residual interaction in the medium gives rise to an overall shift of about $25 \mathrm{MeV}$. The interaction is strongly attractive in the spin-longitudinal ( $\mathrm{LO}$ ) channel due to the attractive $\pi$-exchange interaction, leading to a lowering of the $\Delta$ mass in that channel by about $50 \mathrm{MeV}$. No 
significant shift is, however, observed in the spin-transverse part of the spectrum. Other effects contributing to the shift $(\sim 15 \mathrm{MeV})$ come from the spreading potential which describes the $\Delta$ conversion processes, such as $\Delta+N \rightarrow N+N$. A similar result has also been obtained by other authors. ${ }^{5}$

The calculations are based on the isobar model ${ }^{6,7}$ and DWIA. Denoting the chargeexchange process as $A+a \rightarrow(B+\Delta)+b$, where $A(B)$ and $a(b)$ denote the target (residual nucleus) and projectile (ejectile), respectively, the inclusive cross section is given by

$$
\frac{d^{2} \sigma}{d J_{b}^{\prime} d \Omega_{b}}=\frac{E_{a} E_{b}^{\prime} E_{A} E_{B+\Delta}}{\left(2 \pi \hbar^{2} c^{2} W\right)^{2}} \frac{k_{b}}{k_{a}} R
$$

where $R$ is the strength function defined by

$$
R=\operatorname{Im}[-<\rho|G| \rho>/ \pi]=\operatorname{Im}[-<\rho \mid \psi>/ \pi] \quad(|\psi>\equiv G| \rho>) .
$$

In Eq. (1), $E_{i}$ is the total energy of particle $i(i=a, b, A$, and $B+\Delta), k_{a}\left(k_{b}\right)$ is the wave number of $a(b)$, and $W$ is the total energy in the cf iter of momentum system. $|\rho\rangle$ in Eq. (2) is the doorway state defined as $|\rho\rangle=\left(\chi_{b}^{(-)} \varphi_{b}\left|t_{N N, N \Delta}\right| \chi_{a}^{(+)} \varphi_{a} \varphi_{A}>\right.$, where $\chi_{a}^{(+)}$ $\left(\chi_{b}^{(-)}\right)$is the projectile distorted wave function in the incident (exit) channel, $\varphi_{a}\left(\varphi_{b}\right)$ is the intrinsic wave functions of $a(b)$, and $\varphi_{A}$ is the initial target wave function, and $t_{N N, N \Delta}$ is the effective $N N \rightarrow N \Delta$ transition operator. The round bra $(\mid>$ denotes integration with respect to the projectile coordinates only. Further, $G$ in Eq. (2) is the Green's function that describes the propagation of the $(B+\Delta)$ system and contains all nuclear medium effects, while $|\psi\rangle$ is the wave function of the final $B+\Delta$ system. In the isobar model, ${ }^{6,7} G$ is given as

$$
G=\frac{1}{\omega+i \Gamma_{\Delta} / 2-H_{B}-T_{\Delta}-U_{\Delta}-V_{N \Delta, N \Delta}} .
$$

Here, $\omega$ is the excitation energy of the $(B+\Delta)$ system, $\Gamma_{\Delta}(\omega)$ is the energy dependent free decay width of the $\Delta$, while $T_{\Delta}$ and $U_{\Delta}\left(=V_{\Delta}+i W_{\Delta}\right)$ are the kinetic energy operator and the $\Delta$-nucleus potential, respectively. The $\Delta$-nucleus potential $U_{\Delta}$ includes, as mentioned earlier, an imaginary part that represents the conversion (spreading) effect. $H_{B}$ is the Hamiltonian of nucleus $B$, and $V_{N \Delta, N \Delta}$ is the residual interaction describing the $\Delta N^{-1}$ correlations.

In the numerical calculations, use was made of $U_{\Delta}$ determined from analyses of $\pi$-nucleus scattering. ${ }^{7}$ The $V_{N \Delta, N \Delta}$ was assumed to consist of $\pi$ and $\rho$-exchange potentials with an 
additional short range interaction. ${ }^{8}$ Further, the $t_{N N, N \Delta}$-amplitude was assumed to be simple $\delta$-function type force given as $t_{N N, N \Delta}=g_{N \Delta}^{\prime} J_{\pi N \Delta}\left(\frac{\Lambda_{\pi}^{2}-m_{x}^{2}}{\Lambda_{\pi}^{2}-t}\right)^{2}\left(\vec{\sigma}_{1} \cdot \vec{S}_{2}^{\dagger}\right)\left(\vec{T}_{1} \cdot \vec{T}_{2}^{\dagger}\right)$ with $J_{\pi N \Delta}=4 \pi \hbar c f_{\pi N N} f_{\pi N \Delta} / m_{\pi}^{2} \approx 800 \mathrm{MeV} \mathrm{fm}^{3}, g_{N \Delta}^{\prime}=0.335$. We made this choice since studies of the $t_{N N, N \Delta}$-interaction by means of the $(\vec{d}, 2 \mathrm{p})$-reaction ${ }^{9}$ suggested that $t_{N N, N \Delta}$ is nearly constant in the $(\omega, \vec{q})$-range relevant to the $\Delta$-resonance region, except for the four momentum transfer, $t$, dependence coming from the form factor, and also that $t_{N N, N \Delta}$ should have a simple spin structure as assumed above.

In Fig. 6a, we reproduce our calculated results for the 0 -degree spectra of the ${ }^{12} \mathrm{C}(\mathrm{p}, \mathrm{n})$ reaction at $800 \mathrm{MeV}$ incident energy, in comparison with the experimental data. The theoretical curves were calculated either with or without inclusion of $\Delta$-hole correlations (full and dashed curves, respectively). Also the correlated spin-LO and spin-transverse (TR) cross sections are shown separately. It is seen that the peak position of the spin-LO spectrum is lowered col siderably in energy in comparison with the spin-TR spectrum or with the uncorrelated result. This is due to the attractive $\pi$-exchange interact on in the spin-LO channel. The peak of the uncorrelated spectrum appears at $\omega \approx 320 \mathrm{MeV}$, somewhat lower than the 330 (=370-40) MeV that are expected from the binding energy effect on the $\Delta$ and the $N$. This small shift is due to the conversion (spreading) effect. Fig. 6b shows cross section contributions of various spin states. It is seen that the spectra of lower spin states have their peak at lower $\omega$, showing that the $\Delta N^{-1}$ correlations are most important for these lower spin states.

Similar calculations were also done for the $\left({ }^{3} \mathrm{He}, \mathrm{t}\right)$ reaction, obtaining a similar fit to the data as obtained in the $(p, n)$ reaction. The work reported here was presented at various international meetings. ${ }^{10-16}$. 
1. C. G. Cassapakis et al., Phys. Lett. 63B, 35 (1976)

B. E. Bonner et al., Phys. Rev. C 18, 1418 (1978).

D. A. Lind, Can. J. Phys. 65, 637 (1987).

2. V. G. Ableev et al., Phys. JETP Lett. 40, 763 (1984).

D. Contardo et al., Phys. Lett. 168B, 331 (1986).

3. C. Ellegaard et al., Phys. Rev. Lett. 59, 974 (1987).

4. T. Udagawa, S. W. Hong and F. Osterfeld, Phys. Lett. B245, 1 (1990). (See also 3-14.)

5. J. Delorme and P. A. Guichon, Phys. Lett. B263, 157 (1991).

6. L. S. Kisslinger and W. Wang, Ann. of Phys. 108, 116 (1977)

7. M. Hirata, J. H. Koch, F. Lenz and E. J. Moniz, Ann. of Phys. 120, 205 (1979).

E. Oset, H. Toki and W. Weise, Phys. Rep. 83, 281 (1982).

8. M. R. Anastasio and G. E. Brown, Nucl. Phys. A285, 516 (1977).

9. C. Ellegaard et al., Phys. Lett. B 231, 365 (1989).

10. F. Osterfeld, S.-W. Hong, and T. Udagawa, Proceeding of the International Conference on Selected Topics in Nuclear Structure, Dubna (1989) p.86-94. (See also 3-15.)

11. T. Udagawa, S.-W. Hong, and F. Osterfeld, Proc. of Second LAMPF International Workshop on Pion-Nucleus Double Charge-Exchange, eds. by W. R. Gibbs and M. J. Leitch (World Scientific, 1989), p. 439. (See also 3-16.)

12. S.-W. Hong, F. Osterfeld, and T. Udagawa, Invited talk given at International Workshop on Nuclear Structure and Nuclear Reactions, RIKEN, Japan, eds. by K.-I. Kubo, M. Ishihara, M. Ichimura, and S. Yamaji (World Scientific, 1991), p. 261. (See also 3-17.)

13. F. Osterfeld, S.-W. Hong, and T. Udagawa, Nucl. Phys. A527, (1991) 405c. (See also 3-18.)

14. T. Udagawa, P. Oltmanns, and F. Osterfeld, Proc. of International Conference on Spin and Isospin in Nuclear Interactions, eds. by S. W. Wissink, C. D. Goodman, and G. E. Walker (Plenum Press, New York, 1991), p. 69. (See also 3-19.)

15. Г. Udagawa, F. Osterfeld, P. Oltmanns, and S.-W. Hong, Proc. of the 6-th International Conference on Nuclear Reaction Mechanisms, eds. by E. Gadioli (Universita Deglistudi di Milano, Milano, 1991) p.632. (See also 3-20.)

16. F. Osterfeld, B. Körfgen, P. Oltmanns, and T. Udagawa, Proc. of International Workshop on Meson Production, Interaction and Decay, Krakow, May 7-11, 1991. (See Also 3-21.)

\subsubsection{Exclusive Cross Sections}

The inclusive cross section given by Eqs. (1)-(2) in the previous subsertion 2.2.1 includes contributions from three different processes characterized by the mode of the subsequent decay of the $\Delta$ after its creation. The three different decay modes in question are the quasi-free (qf) decay $\Delta \rightarrow \pi+N$, the decay due to the conversion (spreading) process $\Delta+N \rightarrow N+N$, and the decay resulting from single pion production, $\Delta+N^{-1} \rightarrow \pi$ 
(coherent $\pi$ production). Correspondingly, the strength function $R$ given by Eq. (2) can be split into a sum of three terms

$$
R=\left[<\psi\left|\frac{\Gamma_{\Delta}}{2}\right| \psi>+<\psi\left|-W_{\Delta}\right| \psi>+<\psi\left|-\operatorname{Im}\left(V_{N \Delta, N \Delta}\right)\right| \psi>\right] / \pi .
$$

Fig. 7 shows the exclusive cross section ${ }^{1,2}$ for the qf, conversion, and coherent $\pi$-production events. Note that $W_{\Delta}$ and $V_{N \Delta, N \Delta}$, involved in the conversion and coherent $\pi$-production components in the above expression, resprectively, are ingredients of the nuclear medium that make an important contribution to the shift discussed in the previous subsection 2.2.1. Therefore, comparisons of the predicted exclusive cross sections with the data are expected to provide further tests of our theoretical calculations reported in 2.2.1.

Exclusive cross section data that we can use for such comparisons recently became available ${ }^{3,4}$ through measurements of the ${ }^{12} \mathrm{C},\left({ }^{3} \mathrm{He}, \mathrm{t}\right)$ and ${ }^{12} \mathrm{C}(\mathrm{p}, \mathrm{n})$ reactions in coincidence with $\pi+p, 2 p$ and (single) $\pi$ events. Such data were taken for incident ${ }^{3} \mathrm{He}$ and $p$ energies of $2 \mathrm{GeV}$ and $800 \mathrm{MeV}$, respectively, with the triton and neutron emitted at forward angles. These coincidence cross sections are compared with calculations in Fig. 8, taking the (p,n) reaction case. ${ }^{4}$ In doing this, however, we introduced overall normalization factors of 0.25 , 0.38 and 1.67 for the $\mathrm{qf}$, conversion, and coherent $\pi$ production cross sections, respectively. With these normalization factors, the theoretical curves fit the experimental data fairly well. It is particularly remarkable that the different peak positions for the three cross sections are very well reproduced by the calculations. (A similar result has been obtained also for the ${ }^{12} \mathrm{C}\left({ }^{3} \mathrm{He}, \mathrm{t}\right)$ reaction. See Ref. 2.)

The normalization factors 0.25 and 0.38 introduced for the qf and conversion cross sections, respectively, account partially for the final state absorption of $\pi$ and/or $p$ by the target. (The absorption of $\pi$ in the coherent $\pi$ production process is automatically taken into account in the calculation.) Another source for the normalization factors is that the calculated of and conversion cross sections include besides the $\pi$, and $2 p$ events also the $\pi n$ and $p n$ production processes. The measured $\pi p$ and $2 p$ product on cross sections should therefore be smaller than the cross sections predicted from the first and second term in Eq. (1). Another source for the need of a renormalization of the theoretical cross sections comes from the fact that the measurements of the coincidence cross sections are made with a certain finite acceptance 
angle and energy. (See Refs. 3 and 4 for the details.) In comparing the calculated cross sections with the measured ones, we have to renormalize the former appropriately. This is done by the normalization factors in Fig. 8.

In contrast to the $\pi p$ and $2 p$ coincidence cross sections, the normalization factor 1.67 for the coherent $\pi$ production cross section is larger than unity. This may reflect the fact that the experimental cross section includes not only the contribution from real coherent $\pi$ production events, but also contributions from the qf decay processes in which only the $\pi$ is detected, but the proton is not detected because of the "acceptance" problem of the detection system. The experimental cross section may also include contributions from the qf $\Delta \rightarrow \pi_{T} n$ process. Considering all these factors, the fit obtained in Fig. 8 with the assumed normalization factors seems to be quite significant.

The result of the calculations has indicated that the $\mathrm{qf} \pi p$ and the conversion $2 p$ events are dominated by the spin-TR channel, while the coherent $\pi$ events are dominated by the spin-LO channel. Thus coherent $\pi$ production events are a good source of information on the $\pi$ correlation. A more de riled analysis of the data, i.e., the analyses of the energy and angular distributions of the emitted $\pi$, would be both interesting and important.

1. T. Udagawa, F. Osterfeld, and P. Oltmanns, Proceeding of the LAMPF Workshop on $N-N$ and $N$-Nucleus Scattering held at Los Alamos, to be published. (See also 4-3.)

15. T. Udagawa, F. Osterfeld, P. Oltmanns, and S.-W. Hong, Proc. of the 6-th International Conference on Nuclear Reaction Mechanisms, eds. by E. Gadioli (Universita Deglistudi di Milano, Milano, 1991) p.632. (See also 3-20.)

3. T. Hennino et al., Phys. Lett. B 283, 42 (1992)

4. J. Chiba et al., Phys. Rev. Lett. 67, 1982 (1991).

\subsubsection{Spin-Observables}

Another source of information on the $\pi$ correlations discussed in the previous subsection is in the spin transfer coefficients $C_{X X}$ and $C_{Z Z}$, since these quantities are quite sensitive to the 
correlation. Defining the spin-LO and spin-TR strength functions, $R_{L}$ and $R_{T}$, respectively, as

$$
R_{i}=\operatorname{Im}\left[-<\rho_{i}|G| \rho_{i}>/ \pi\right] \quad(i=L \text { or } T)
$$

where

$$
\left|\rho_{i}>=\hat{O}_{i}\right| \varphi_{A}>
$$

with

$$
\hat{O}_{L}=e^{i \vec{q} \cdot \vec{r}}(\vec{S} \cdot \hat{q}) \hat{T}_{-}
$$

and

$$
\hat{O}_{T}=e^{i \vec{q} \cdot \vec{r}}(\vec{S} \times \hat{q}) \hat{T}_{-},
$$

the spin transfer coefficients can be evaluated as

$$
\begin{gathered}
C_{X X}=\frac{-\left|R_{L}\right|^{2}}{\left|R_{L}\right|^{2}+2\left|R_{T}\right|^{2}}, \\
C_{Z Z}=\frac{\left|R_{L}\right|^{2}-2\left|R_{T}\right|^{2}}{\left|R_{L}\right|^{2}+2\left|R_{T}\right|^{2}} .
\end{gathered}
$$

From these equations, the sensitivity of the spin transfer coefficients to the $\pi$ correlations in $R_{L}$ is obvious.

In Fig. 9, we present the calculated spin transfer coefficients $C_{X X}$ and $C_{Z Z}$ for the $p(\vec{p}, \vec{n}) \Delta^{++}$reaction at the proton incident energy of $800 \mathrm{MeV}$ in comparison with the data. ${ }^{1}$ These data are found to be well described by our calculations using the $t_{N N, N \Delta \text {-operator }}$ given by Eq. (5). This confirms that the spin structure of the $t_{N N, N \Delta}$-operator used in our study $^{2}$ of the medium effects on $\Delta$ is realistic.

The calculated spin transfer coefficients for the $800 \mathrm{MeV}^{12} \mathrm{C}(\mathrm{p}, \mathrm{n})$ reaction are shown in Fig. 10. Here the observables $C_{X X}$ and $C_{Z Z}$ calculated with inclusion of $\Delta N^{-1}$ correlations (full curves) are compared with the uncorrelated results (broken curves). A large effect due to the correlations is seen in both quantities It is thus highly desirable to measure the coefficients $C_{X X}$ and $C_{Z Z}$.

We remark that at Saturne in Saclay an experiment with similar motivation for seeing the spin-LO correlation effect in nuclei was performed. In this experiment the tensor analyzing power in the ${ }^{12} \mathrm{C}(\vec{d}, 2 \mathrm{p})$ reaction at $E_{l a b}=2 \mathrm{GeV}$ was measured. We have analyzed these data, 
finding that values measured at $3-6^{\circ}$ could be explained fairly well by the calculations, but the data at the extreme forward angles ( $0^{\circ}$ data particularly) were not reproduced very well, particularly in the excitation energy region where the pion correlations play an important role, i.e., at the lower excitation energy region. In Fig. 11, we present the results of these calculations, including the fit to the inclusive cross sections. We believe that the problems we find in describing the data at forward angles and lower excitation energies are due to cross section contributions from other reaction mechanisms, e.g., one- and two-step nucleonknockout and various other two-step mechanisms. The fact that both tensor analyzing power and inclusive cross section data consistently defy explanation seems to support our view.

Just as in the $(\vec{d}, 2 p)$ reaction, contributions from different reaction mechanisms other than one-step $\Delta$ excitation might also contribute to the $(p, n)$ reaction. It seems likely, though, that these processes should have, if any at all, less effect in the $(\mathrm{p}, \mathrm{n})$ than in the $(\vec{d}, 2 p)$ reaction. This is particularly true for the lower $\omega$ energy side of the $\Delta$ resonance, as the observed $(p, n)$ spectra seem to show that the reaction is dominated here by the one-step $\Delta$ excitation process. The work described above was presented ${ }^{3}$ at the Telluride Conference held on 11-15, March 1991.

1. G. Glass et al., Phys. Lett. B129, 27 (1983).

2. T. Udagawa, S. W. Hong and F. Osterfeld, Piys. Lett. B245, 1 (1990). (See also 3-14.)

3. T. Udagawa, P. Oltmanns, and F. Osterfeld, Spin and Isospin in Nuclear Interactions, eds. by S. W. Wissink, C, D. Goodman, and G. E. Walker (Plenum Press, New York, 1991), p. 111. (See also 3-19.) 


\subsection{Nuclear S'sructure}

We have proposed recently a new approach to continuum RPA calculations. In this approach, we construct a coupled-channel (CC) integral equation for a set of radial wave functions $\lambda_{p h}(r)$ for the particle $p$ excited from a hole orbit $h$. We call $\lambda_{p h}(r)$ the correlated source functions. An important feature of $\lambda_{p h}(r)$ is that they are localized. We are thus able to solve the $\mathrm{CC}$ integral equation very efficiently by applying the Lanczos method. For solving the $\mathrm{CC}$ equation for the $2^{+}$channel in ${ }^{208} \mathrm{~Pb}$, which has a dimension of 149 , for instance, we needed only 10 iterations to reach convergence. This means that we were able to reduce the computation time by at least an order of magnitude. The method has thus enabled us to perform such studies of the nuclear medium effects on $\Delta$ as described in 2.2.

Another advantage of the method is that it enables us to calculate contributions from particle-hole (ph) components involved in the total strength function. This means that with this method we are able to calculate the particle emission decay probabilities and also a variety of coincidence cross sections. In fact, we have successfully applied the method to calculate the particle decay widths for the giant monopole response states. In what follows, we describe the work done on the application to the giant resonances and improvement of the method.

\subsubsection{Random-Phase-Approximation Calculations of Nuclear Response in the Continuum}

In this subsection, we first present the results of the application to the $2^{+}$strength function, $S$, in the giant quadrupole resonance (GQR) region. ${ }^{1}$ In Ref. 1 , the formulation of the method of the calculation has been given. As already noted in the introductory remark, the essence of the approch is the use of the Lanczos method. ${ }^{2}$

Fig. 12 shows the calculated distributions $F(E)$ of the energy-weighted quadrupole transition strength in unit of the total sum rule, i.e.,

$$
F(E)=\left[E S(E) / \int_{0}^{\infty} E^{\prime} S\left(E^{\prime}\right) d E^{\prime}\right] \times 100
$$

The corresponding experimental data are also presented by a histogram with a $1 \mathrm{MeV}$ bin. These experimental data are those deduced from hadronic inelastic scattering experiments. ${ }^{3-4}$ 
As seen, the calculations reproduce the observed distributions fairly well. The calculated peak energies $\left(E_{R}\right)$ and widths $(\Gamma)$ of the GQR are $E_{R}=21.2,16.8$ and $11.1 \mathrm{MeV}$ and $\Gamma=6.4,4.4$ and $1.8 \mathrm{MeV}$ for ${ }^{16} \mathrm{O},{ }^{40} \mathrm{Ca}$ and ${ }^{208} \mathrm{~Pb}$, respectively. These are compared with the experimental values of $E_{R, e x p}=21,18$ and $11 \mathrm{MeV}$, and $\Gamma_{\text {exp }}=7.5,4.0$ and $2.0 \mathrm{Mel}$, respectively.

The above calculations included effects of the damping of the excited particle by means of the imaginary part included in its mean field, but ignored the effects for the hole states as well as the interference between the particle and hole states. In spite of this, the calculation could reproduce fairly well the observed width $\Gamma_{\text {exp. }}$. This suggests that the neglected effects are small as expected from the fact that the dominant contribution to $S$ comes from ph components, whose hole states are in the last occupied shell, and that the contribution to the spreading from such hole states may be small. It might also be possible that this is achieved because the two contributions neglected are cancelling one another. ${ }^{5}$

The calculations predicted $S^{\dagger} / S$ at the peak energy to be $12 \%, 8 \%$ and $5 \%$, respectively, for ${ }^{16} \mathrm{O},{ }^{40} \mathrm{Ca}$ and ${ }^{208} \mathrm{~Pb}$. This result shows that the decay should be dominated by the damping, which agrees with the data taken so far, although there are no data which would permit a quantitative comparison with the calculated results.

The calculations reported above were all done by assuming the mass multipole field as the external force. We recently extended the calculations to use a more realistic force, i.e., the M3Y force of Bertsch et al. ${ }^{6}$ for ( $\left.\mathrm{p}, \mathrm{p}^{\prime}\right)$ reactions. With the M3Y force, the source function was calculated by means of DWIA. Such calculations were reported at two international meetings. ${ }^{78}$

1. T. Udagawa and B. T. Kim, Phys. Rev. C 40, 2271 (1989). (See also 3-21.)

2. R. R. Whitehead, A. Watt, B. J. Cole and I. Morrison, Advances in Nuclear Physics, Vol.9, p.123 (1977).

3. K. T. Knoepfle et al., Phys. Rev. Lett. 35,779 (1975).

4. Y.-W. Lui et al., Phys. Rev. C $\underline{24}, 884$ (1981).

J. Lisantti et al., to be published.

5. G. F. Bertsch, P. F. Bortignon and R. A. Broglia, Rev. Mod. Phys. 55, 287 (1983).

G. Co, K. F. Quader, R. D. Smith, and J. Wambach, Nucl. Phys. A485, 61 (1988).

6. C. F. Bertsch, J. Borysowicz, H. Mcmanus and W. G. Love, Nucl. Phys. A284, 399 (1977). 
7. B. T. Kim and T. Udagawa, Proceedings of the 5th French-Japanese Symposium on Nuclear Physics, ed. by K. Shimizu and O. Hashimoto, p.270 (1989). (See also 3-23.) 8. B. T. Kim and T. Udagawa, Chin. Jour. of Phys. 29, 431 (1991). (See also 3-24.)

2.3.2 Continuum Random-Phase-Approximation Study of Direct Decay of Giant Monopole Resonances in ${ }^{90} \mathrm{Zr}$ and ${ }^{208} \mathrm{~Pb}$

As a continuation, such continuum RPA calculations as described in 2.3.1 were made ${ }^{1}$ of particle emission widths from the giant monopole resonances (GMR) in ${ }^{90} \mathrm{Zr}$ and ${ }^{208} \mathrm{~Pb}$. As emphasized in the introductory remarks, our method can retain the particle-hole (ph) information, which makes it possible to evaluate contributions from various particle-hole (ph) components. In fact, the strength function $S(E)$ can be decomposed into a sum of the ph components $S_{p h}(E)$, as

$$
S=\Sigma_{p h} S_{p h}
$$

$S_{p h}$ can further be decomposed into two parts $S_{p h}^{l}$ and $S_{p h}^{\dagger}$, i.e., the damping (spreading) and direct particle decay parts, respectively. In terms of $S_{p h}^{1}$, the partial width $\Gamma_{h}^{\dagger}$ of the GMR may be expressed as

$$
\Gamma_{h}^{\dagger}=\Gamma \int_{E_{R}-\Delta E}^{E_{R}+\Delta E} \Sigma_{p} S_{p h}^{\dagger}(E) d E / \int_{E_{R}-\Delta E}^{E_{R}+\Delta E} S(E) d E
$$

where $E_{R}$ and $\Gamma$ are the energy and the width of the resonance, respectively. The values of $E_{R}$ and $\Gamma$ may be extracted from numerically calculated spectra of $S^{\prime}(E)$. $\Delta E$ defines the range of the integration involved, for which we choose as $\Delta E=2 \mathrm{MeV}$. With this choice for $\Delta E$ chosen value, the integration in $\mathrm{Eq}$. (2) can cover the entire region of the resonance.

Numerical calculations were performed ${ }^{2}$ for $S, S_{p h}^{l}$ and $\Gamma_{h}^{1}$ with two different ph interactions, i.e., those of Shlomo and Bertsch ${ }^{3}$ (SB) and of Ring and Speth (RS). ${ }^{4}$ In all the calculations, the strengths of the ph interaction were adjusted so as to reproduce the observed resonance energy $E_{R}$.

Fig.13 shows the calculated distributions $F(E)$ of the energy-weighted monopole transition strength defined as Eq. (1) of 2.3.1. The corresponding experimental data, deduced 
Table 1: Calculated and experimental $\Gamma_{h}^{\dagger}$-values

\begin{tabular}{lcccc} 
Nucleus & hole & $\Gamma_{h}^{\text {exp }}(\mathrm{keV})$ & $\Gamma_{h}^{\dagger S B}(\mathrm{keV})$ & $\Gamma_{h}^{\dagger R S}(\mathrm{keV})$ \\
& & & & \\
\hline${ }^{208} \mathrm{~Pb}$ & $p_{1 / 2}$ & - & 5 & 19 \\
& $f_{5 / 2}$ & $<35$ & 10 & 63 \\
& $p_{3 / 2}$ & 75 & 16 & 43 \\
& $i_{13 / 2}$ & 75 & 8 & 18 \\
& $f_{7 / 2}$ & $<140$ & 20 & 102 \\
& $h_{9 / 2}$ & - & 6 & 1 \\
& & & & \\
${ }^{90} \mathrm{Zr}$ & $g_{9 / 2}$ & 150 & 45 & 56 \\
& $p_{1 / 2}$ & & 20 & 59 \\
\hline
\end{tabular}

from the measured $\alpha$-particle inelastic scattering cross sections ${ }^{5,6}$ are also presented there by histograms with a $1 \mathrm{MeV}$ bin.

As seen, the calculated $F(E)$ fits the experimental data fairly well. From the calculated spectra, one can deduce the widths of $\Gamma^{c a l}=3.0$ and $2.8 \mathrm{MeV}$ for ${ }^{90} \mathrm{Zr}$ and ${ }^{208} \mathrm{~Pb}$, respectively. These values agree very well with the experimental values of $\Gamma^{e x p}=3.0$ and $2.5 \mathrm{MeV} .^{5,6}$ It should be remarked at this stage that the calculated F's with two different ph interaction are almost identical. This is not the case, however, for $\Gamma_{h}^{1}$, as will be seen below.

In Table 1, we summarize the calculated and experimental values of $\Gamma_{h}^{\dagger}$ for neutron emission from various hole states $h$ in ${ }^{207} \mathrm{~Pb}$ and ${ }^{89} \mathrm{Zr} . \Gamma_{h}^{\mid S B}$ and $\Gamma_{h}^{\dagger R S}$ shown there are those obtained by using the SB and RS ph interactions, respectively. Although a large effort has been made in extracting the experimental values, the best values available now, particularly for ${ }^{208} \mathrm{~Pb}$, are still upper limit values. This results from the fact that to extract these values from the measured (coincidence cross section) data is extremely difficult, because in doing so one has to subtract from the measured data large background and statistical components, neither of which are very well known. The values listed in Table 1 are those determined by assuming the lower limit of the statistical components, and therefore should be viewed as an upper limit.

As seen in Table 1, the two theoretical values are fairly different from each other, indicating the sensitivity of $\Gamma_{h}^{\text {tcal }}$ to the ph interaction. Such a sensitivity was noticed earlier in Ref. 9. 
The result that the theoretical calculations are sensitive to the ph interaction implies that we may use the data for $\Gamma_{h}^{\text {eexp }}$ in testing the ph interaction. At this moment, however, it is not possible to make such a test, since the data available now are only the upper limit values. It is thus highly desirable that more accurate data be taken. Note that $\Gamma_{h}^{\text {ical }}$ is generally smaller than $\Gamma_{h}^{\text {lexp }}$. This is particularly true for the case for $\Gamma_{h}^{\dagger S B} ; \Gamma_{h}^{\dagger S B}$ is smaller by a factor of $4-8$ in ${ }^{208} \mathrm{~Pb}$ and 3 in ${ }^{90} \mathrm{Zr}$ than is $\Gamma_{h}^{\text {lexp }}$. The situation is better in $\Gamma_{h}^{\dagger R S}$, but still underestimates $\Gamma_{h}^{\text {exp }}$ (except the $f_{7 / 2}$ case).

In the study reported above, we included the damping of the excited particles, but not of the holes. We also ignored the interference effect, whose importance has been indicated in recent studies. ${ }^{10}$ The neglect of the damping of the hole states may be justified, since the holes of the main ph-components in GMR are those in the uppermost occupied shells, whose spreading width (damping) is rather small.

Microscopic calculations done in the past have indicated the importance of interference effects, but such calculations still significantly underestimate the width of the GMR. ${ }^{10}$ One possible source may be traced to the fact that the calculations introduce a rather dramatic approximation to replace real final nuclear states by pure $2 \mathrm{p}-2 \mathrm{~h}$ states, into which the collective monopole state damps. Such an approximation leads to a large interference effect, which cancels out contributions from the damping of the particle and the hole. It is highly probable that the interference effects will be reduced if the approximation is removed; a strong mixing of $2 \mathrm{p}-2 \mathrm{~h}$ and more complicated states with a random phase nature will then be induced, which in turn causes cancellations between contributions from various $2 \mathrm{p}-2 \mathrm{~h}$ components, thereby reducing the net contribution. At this moment, it is very difficult to confirm what was argued above by carrying out a realistic calculation. In the present study, we have adopted a phenomenological approach, neglecting the effect of the hole damping and the interference for the reason, discussed above, but treating the effect of the damping of the excited particle in a realistic manner. The successes obtained in the present study and also in our previous study ${ }^{2}$ support our view, though much has to be ciune before reaching a more concrete understanding of the problem.

The work described in this and the previous subsections was summerized at the international workshop on spectroscopy and structure of molecules and nuclei, held at Tallahassee, 
March, 1992.11

1. T. Udagawa and B. T. Kim, Phys. Lett. B 230, 6 (1989). (See also 3-25.)

2. T. Udagawa and B. T. Kim, Phys. Rev. C 40, 2271 (1989). (See also 3-22.)

3. S. Shlomo and G. F. Bertsch, Nucl. Phys. A243, 507 (1975).

4. P. Ring and J. Speth, Phys. Lett. B44, 477 (1973).

5. S. Brandenburg et al., Nucl. Phys. A466, 29 (1987).

6. C. M. Rozen, D. H. Youngblood, J. D. Bronson, Y.-W. Lui, and U. Garg, Phys. Rev. C 21, $1252(1980)$.

7. Wim Borghols, Ph. D. Thesis (Groningen, 1988). (Unpublished)

8. S. Brandenburg et al., to be published in Phys. Rev. C 39, 2448 (1989).

9. A. Bracco, J. R. Beene, N. Van Giai, P. F. Bortignon, F. Zardi and R. A. Broglia, Phys. Rev. Lett. 60, 2603 (1988).

10. See, for instance, the following review articles;

G. F. Bertsch, P. F. Bortignon, and R. A. Broglia, Rev. Mod. Phys. 55, 287 (1983);

J. Wambach, Rep. Prog. Phys. 51, 989 (1988).

11. T. Udagawa, to be published. (See 4-4.)

2.3.3 Random Phase Approximation Calculations of the Nuclear Response in the Continuum Using a Nuclear Matter G-Matrix

Most calculations of the nuclear response which use the continuum random phase approximation (CRPA), which is the subject of our work described in the previous subsections 2.3.1 and 2.3.2, have thus far used a $\delta$-force, for instance the Skyrme force, for the residual particle-hole(ph) interaction, since zero-range interactions greatly simplify the numerical calculations. It is natural, however, to try to include realistic finite range residual interactions in CRPA calculations for the same reasons that extensive efforts have been made to use realistic effective interactions in calculating the properties of the low-lying collective states of nuclei. An effort ${ }^{1}$ has thus recently been devoted to extend our CRPA approach ${ }^{2}$ to handle the case where in addition to the direct part, the exchange part of the effective ph interaction is finite range in character. Shigehara, et al. ${ }^{3}$, recently computed the spin-isospin response in the quasielastic region using a nuclear matter finite-range G-matrix, including a full finite range treatment for both the direct and exchange contributions to the ph residual interaction. A limitation to their formalism is that because they directly compute the nuclear 
response function, as opposed to solving the CRPA equations, they can only calculate the singles cross-section of the scattered particle.

We first applied the method to the lowest $3^{-}$state for ${ }^{16} \mathrm{O}$. This case is interesting since it is well known that a discrete RPA calculation with the G-matrix results in an excitation energy and $B(E 3)$ value that agrees nicely with the experimental data ${ }^{4}$. We found that the use of the G-matrix in the finite range CRPA calculation caused the lowest $3^{-}$state to become unstable. In order to obtain a stable RPA result with an excitation energy near the experimental energy $(6.13 \mathrm{MeV})$ and $B(E 3)$ value $\left(1470 \mathrm{e}^{2} \mathrm{fm}^{6}\right)$ we found it necessary to reduce the overall strength of the $\mathrm{G}$-matrix by $19 \%$ for the exchange part. This suggests that the coupling to the continuum plays a very important role. In addition we also applied the method to the $3^{-}$in ${ }^{40} \mathrm{Ca}$, finding it necessary to reduce the ph strength for the exchange term by $37 \%$.

We then applied the method to calculate the $2^{+}$quadrapole strength functions for ${ }^{16} \mathrm{O}$ and ${ }^{40} \mathrm{Ca}$ in the giant resonance region. We used the same renormalized ph interaction that was obtained in the $3^{-}$calculation for each nucleus. The necessity to introduce a renormalized ph interaction is consistent with the observation by previous researchers that using only the standard RPA in a discrete basis with the G-matrix for the low-lying collective states is inadequate because the ph correlations become unphysically large and requires that either one modifies the effective interaction(say by the addition of screening terms) or go to higher $\mathrm{RPA}^{5-6}$.

We studied the validity of the zero-range approximation of the exchange matrix element; use of the approximation pushed the quadrapole strength far below $10 \mathrm{MeV}$. As pointed out by Shigehara, et $a l .^{4}$, the exchange part of the Nakayama nuclear matter G-matrix is strongly momentum dependent, particularly for the $T=S=0$ channel, thus the delta-function approximation is poor. The final remark is that the ratio of $S^{l}$ to $S$ at the peak resonance energy is .26 and .11 for ${ }^{16} \mathrm{O}$ and ${ }^{40} \mathrm{C}$ a respectively. Comparing these results to those obtained in Ref. 2 (.12 and .08). we observe a significant difference for the ${ }^{16} \mathrm{O}$ case. The large emission strength for ${ }^{16} \mathrm{O}$ is probabiy due to the peak resonance occuring at an excitation energy 2.5 $\mathrm{MeV}$ greater than the peak resonance energy obtained in Ref. 2, although this point needs further clarification. 
1. D. Knobles and 'T. Udagawa, Nucl. Phys. A533, 189 (1991) (See also 3-26.)

2. T. Udagawa and B.T. Kim, Phys. Rev. C 40, 271 (1989).

3. T. Shigehara, K. Shimizu, and A. Arima, Nucl. Phys. A492, 388 (1989).

4. K. Nakayama, S. Krewald, J. Speth, and W.G. Love, Nucl. Phys. A431, 419 (1984).

5. M.G.E. Brand, K. Allaart, and W.H. Dickhoff, Phys. Lett. B 214, 483 (1988).

6. S. Drozdz, S. Nishizaki, J. Speth, and J. Wambach, To be published. 


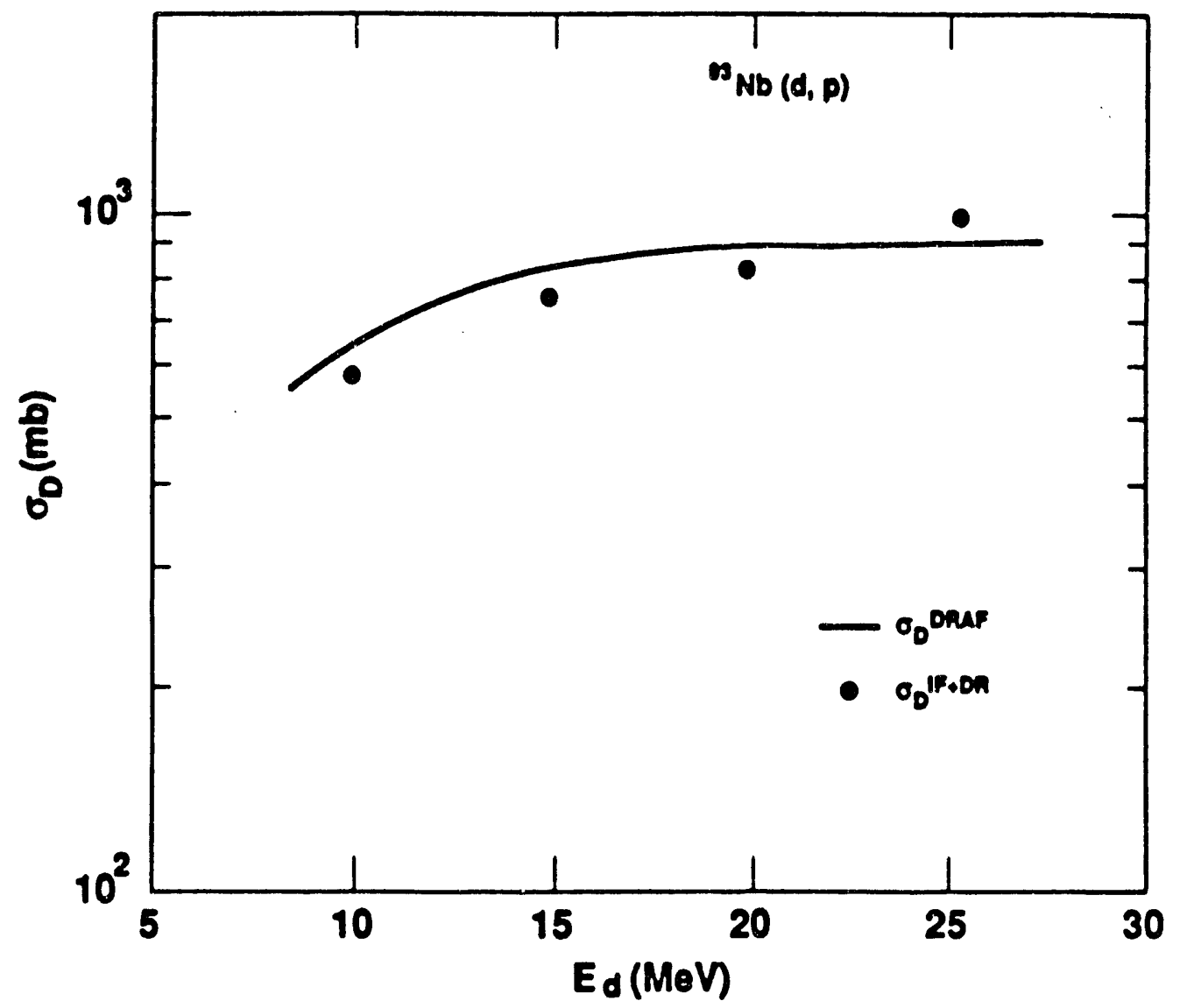

Fig. 1 

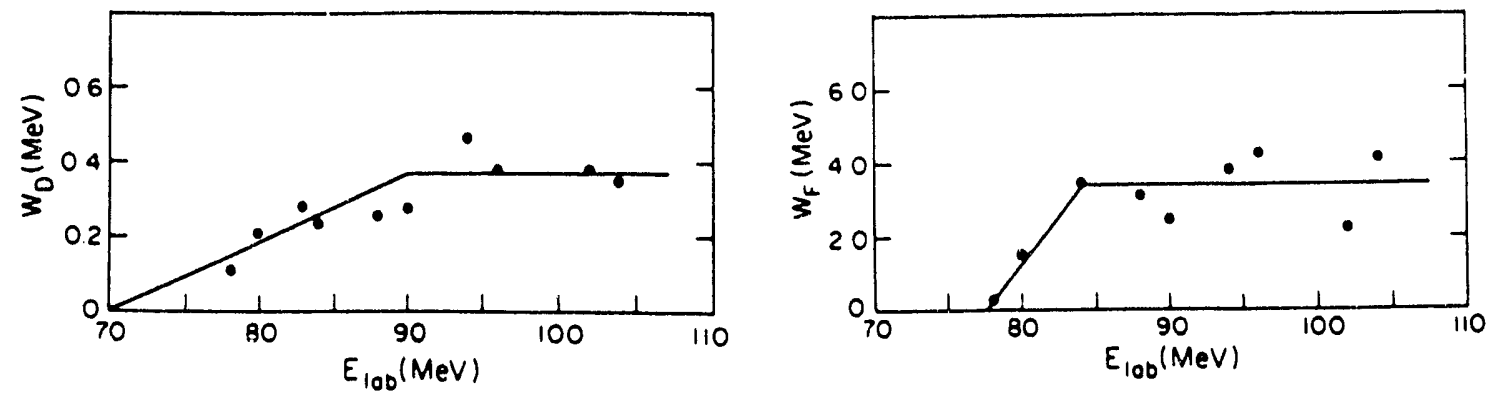

Fig. 2

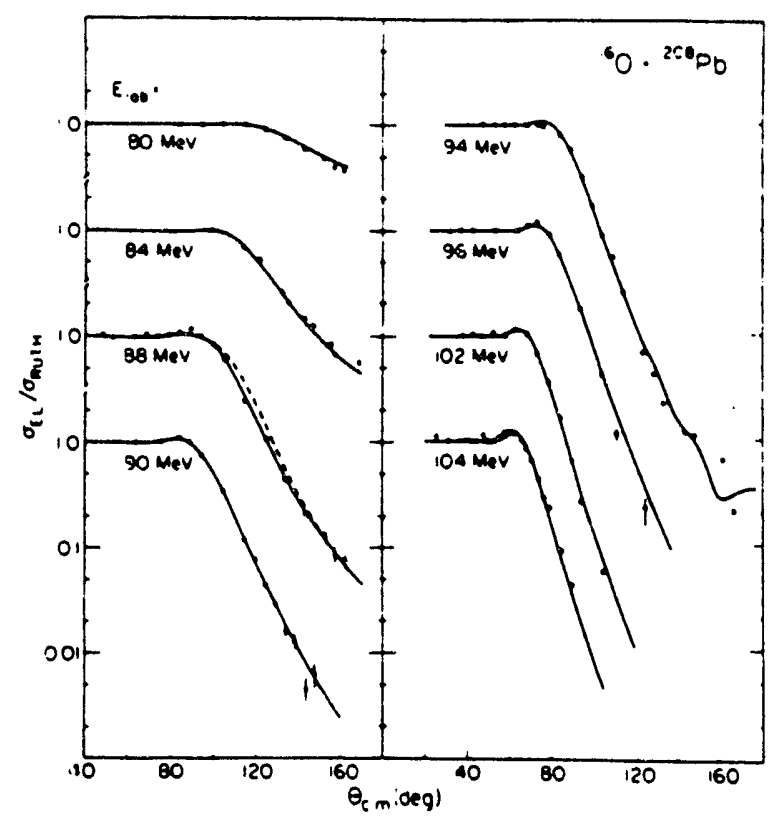

Fig. 3

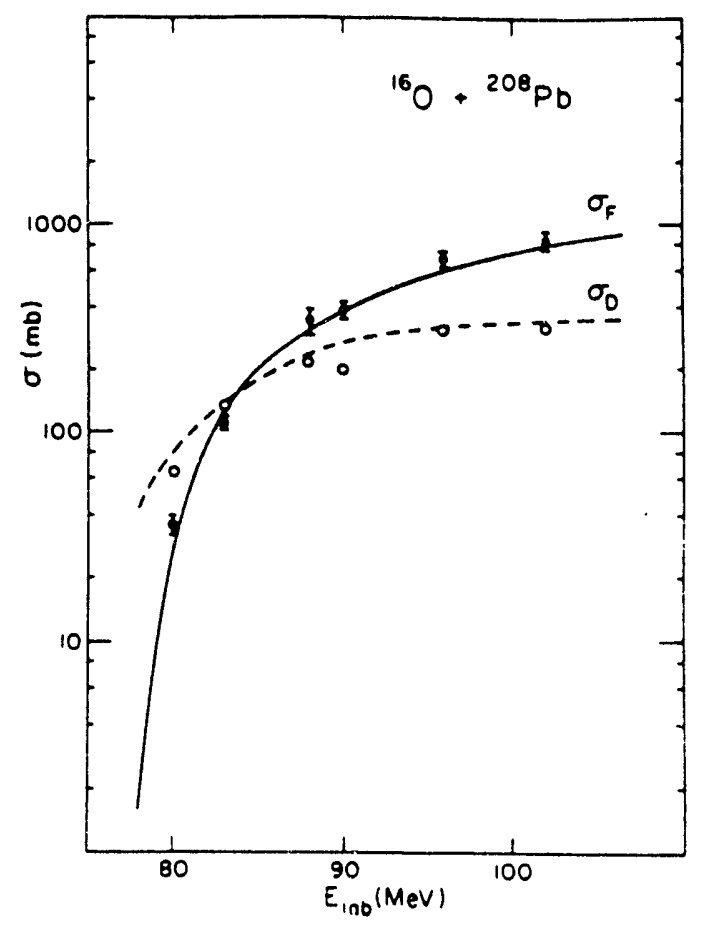

Fig. 4 

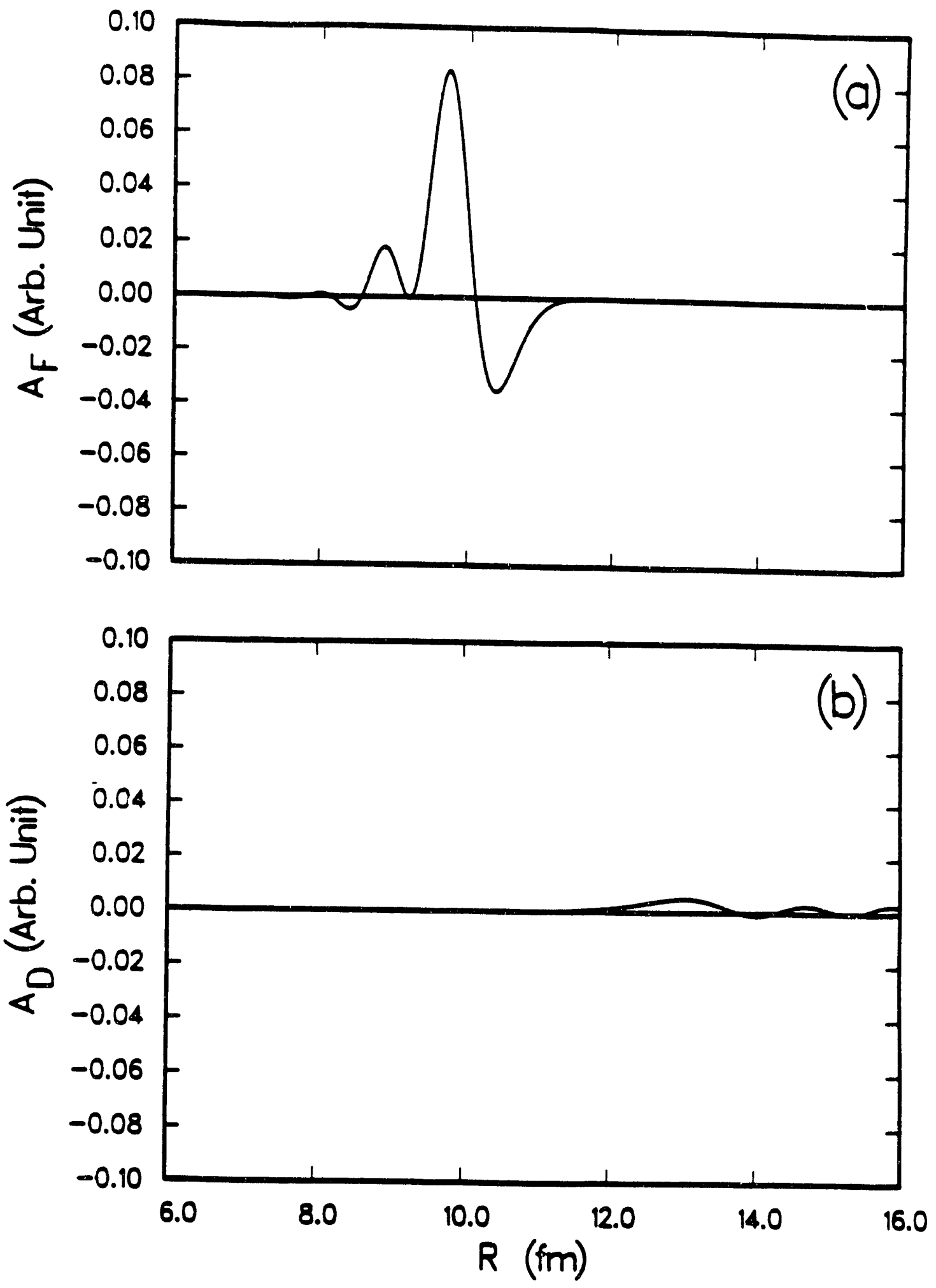

Fig. 5 


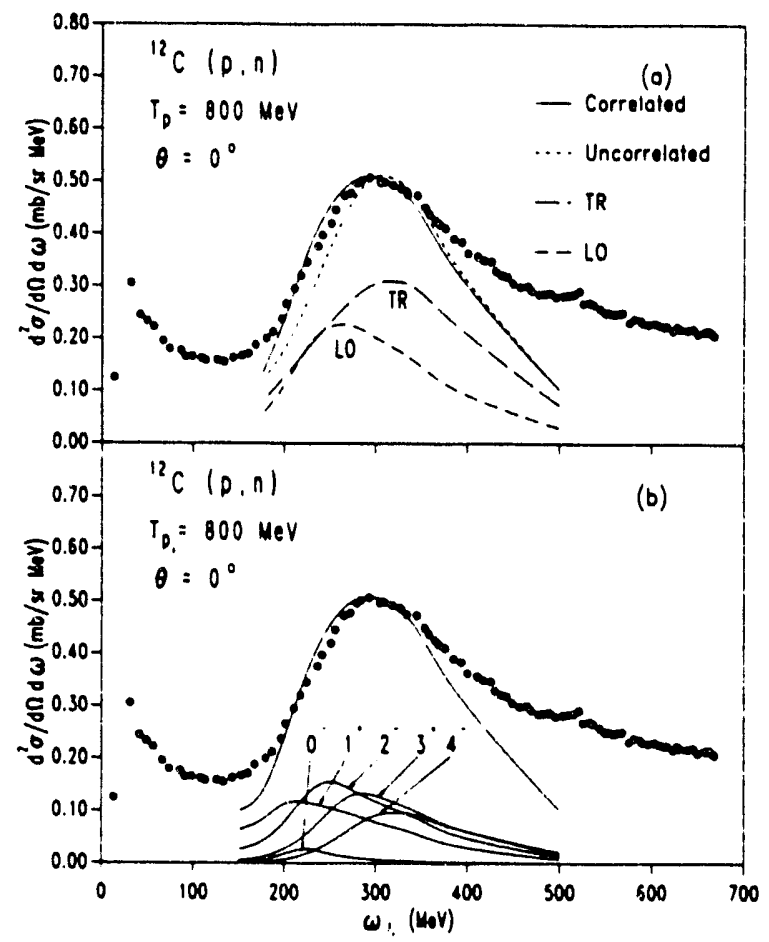

Fig. 6 


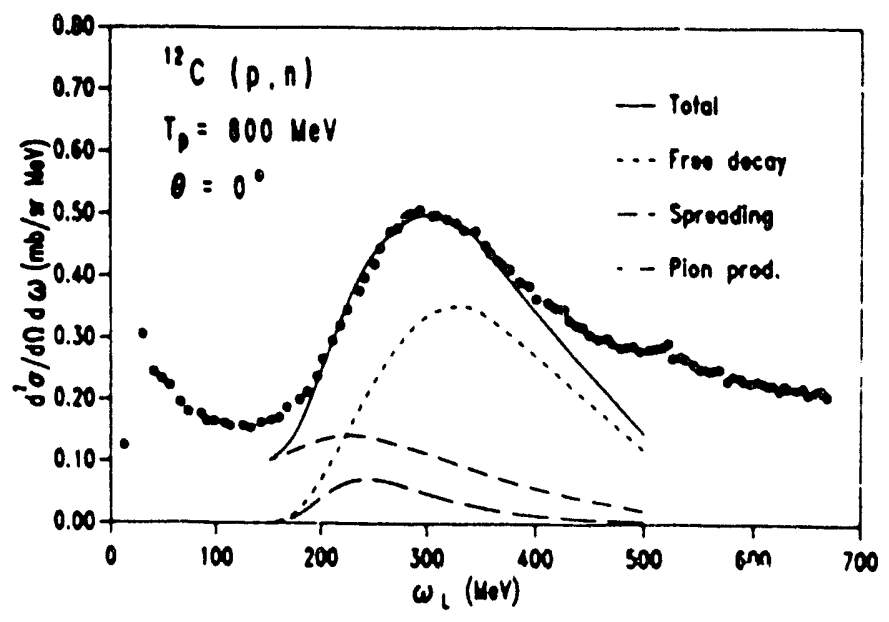

Fig. 7

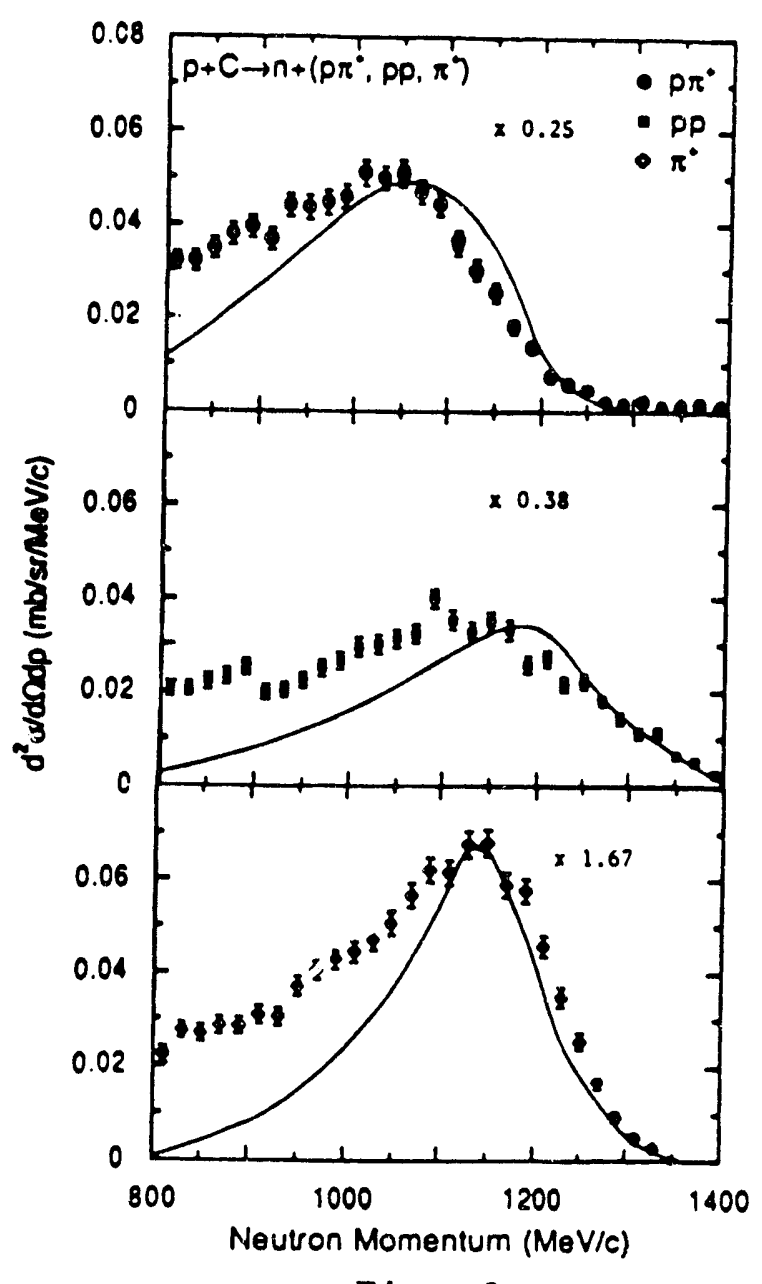

Fig. 8 


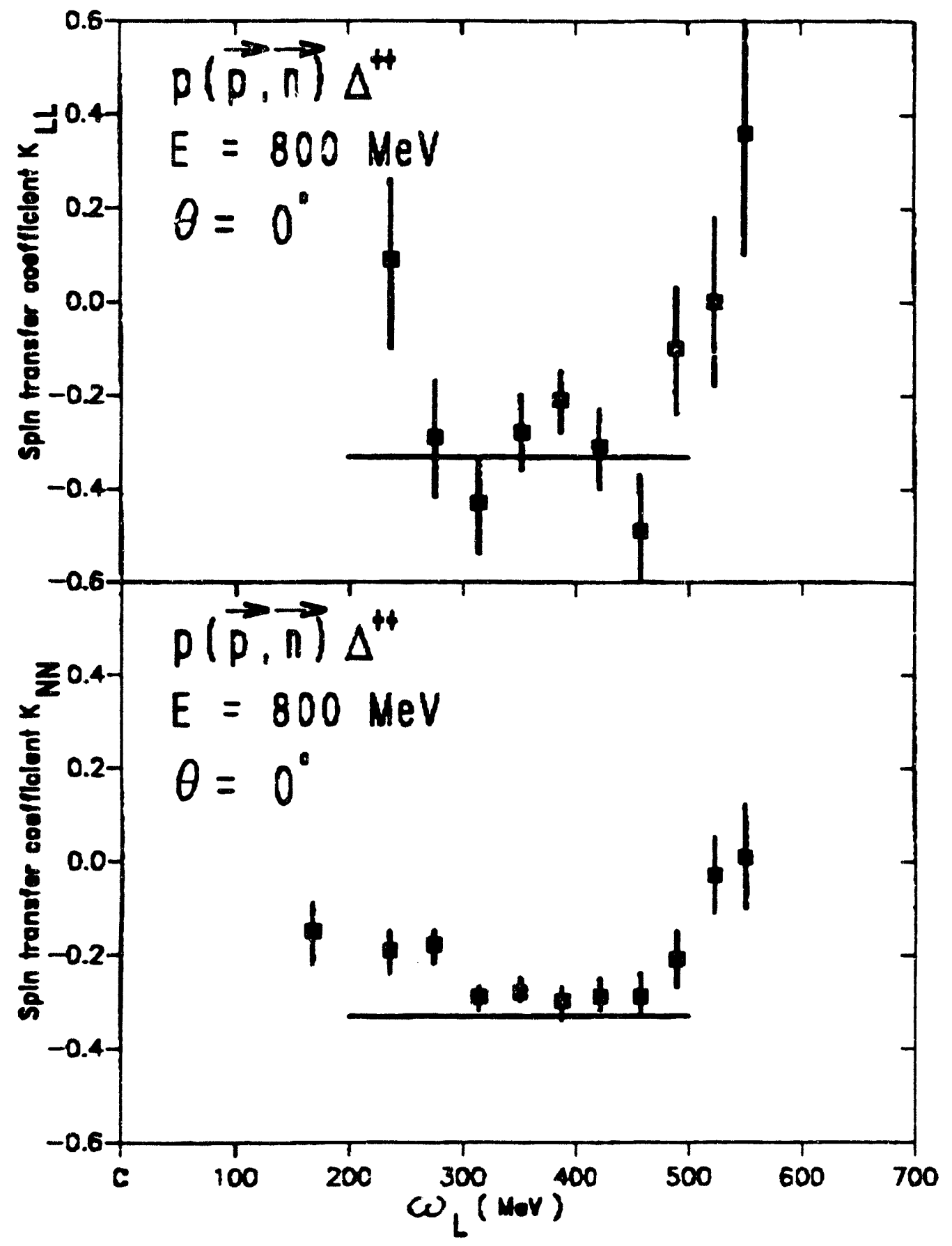

Fig. 9 


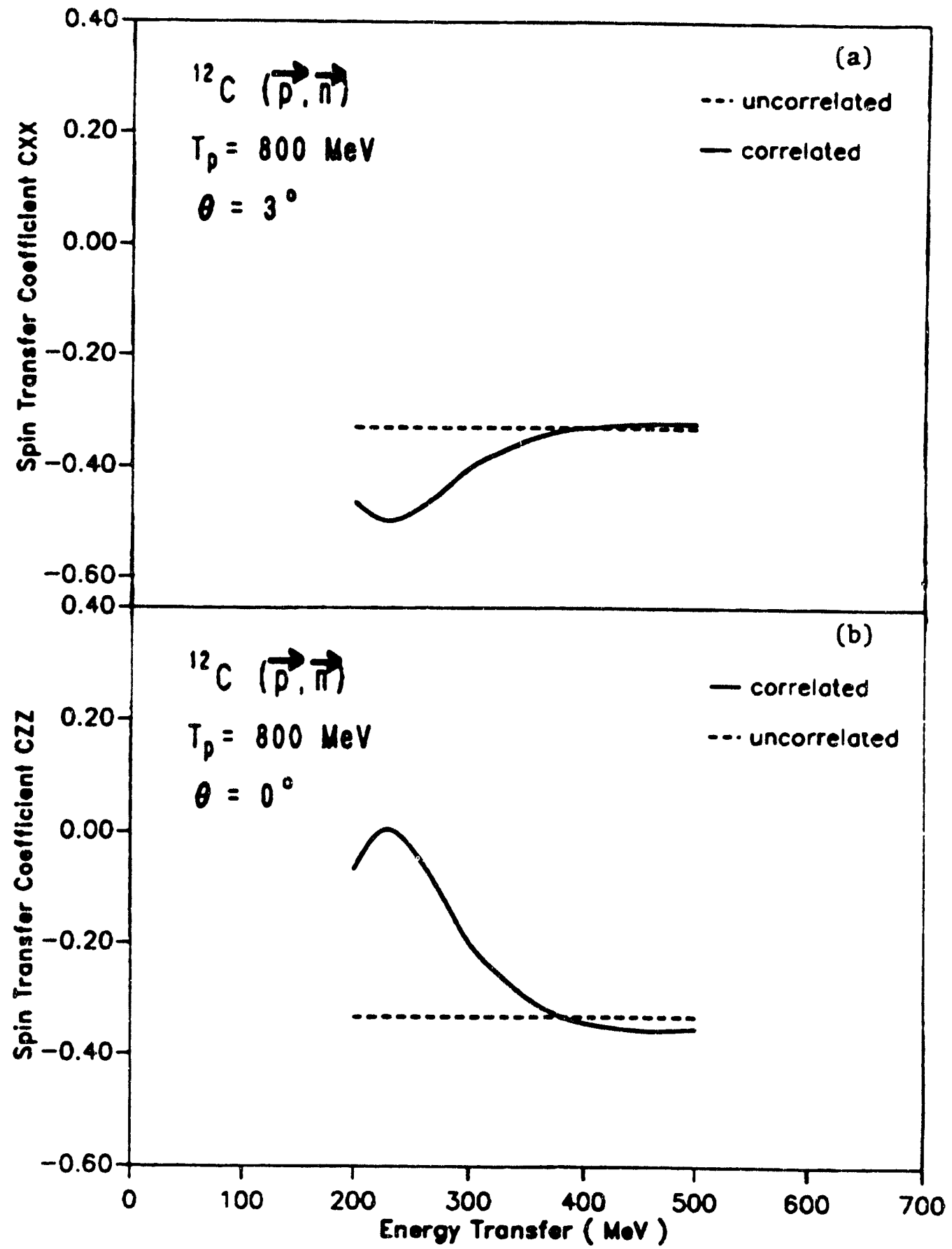

Fig. 10 

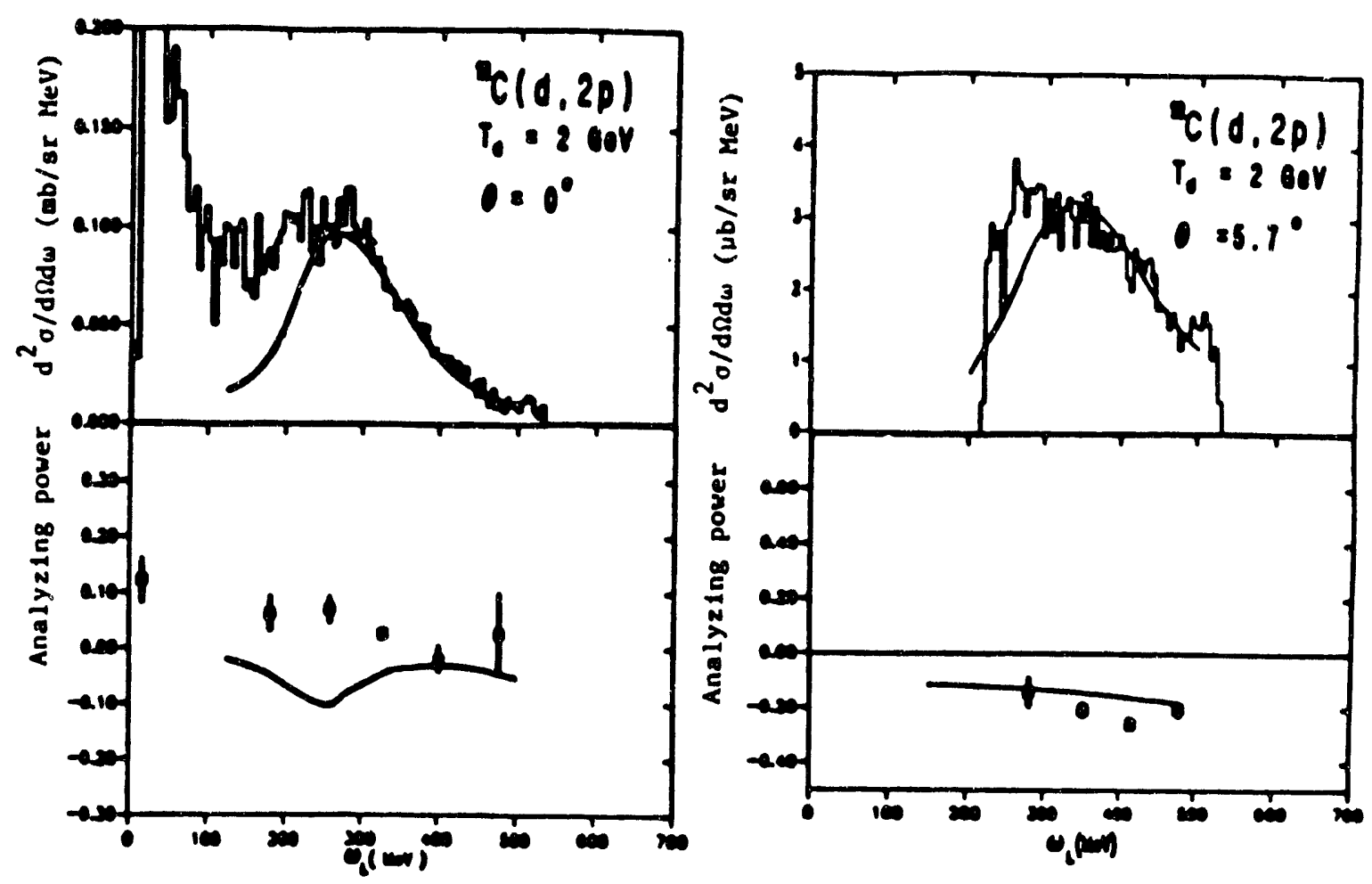

Fig. 11 

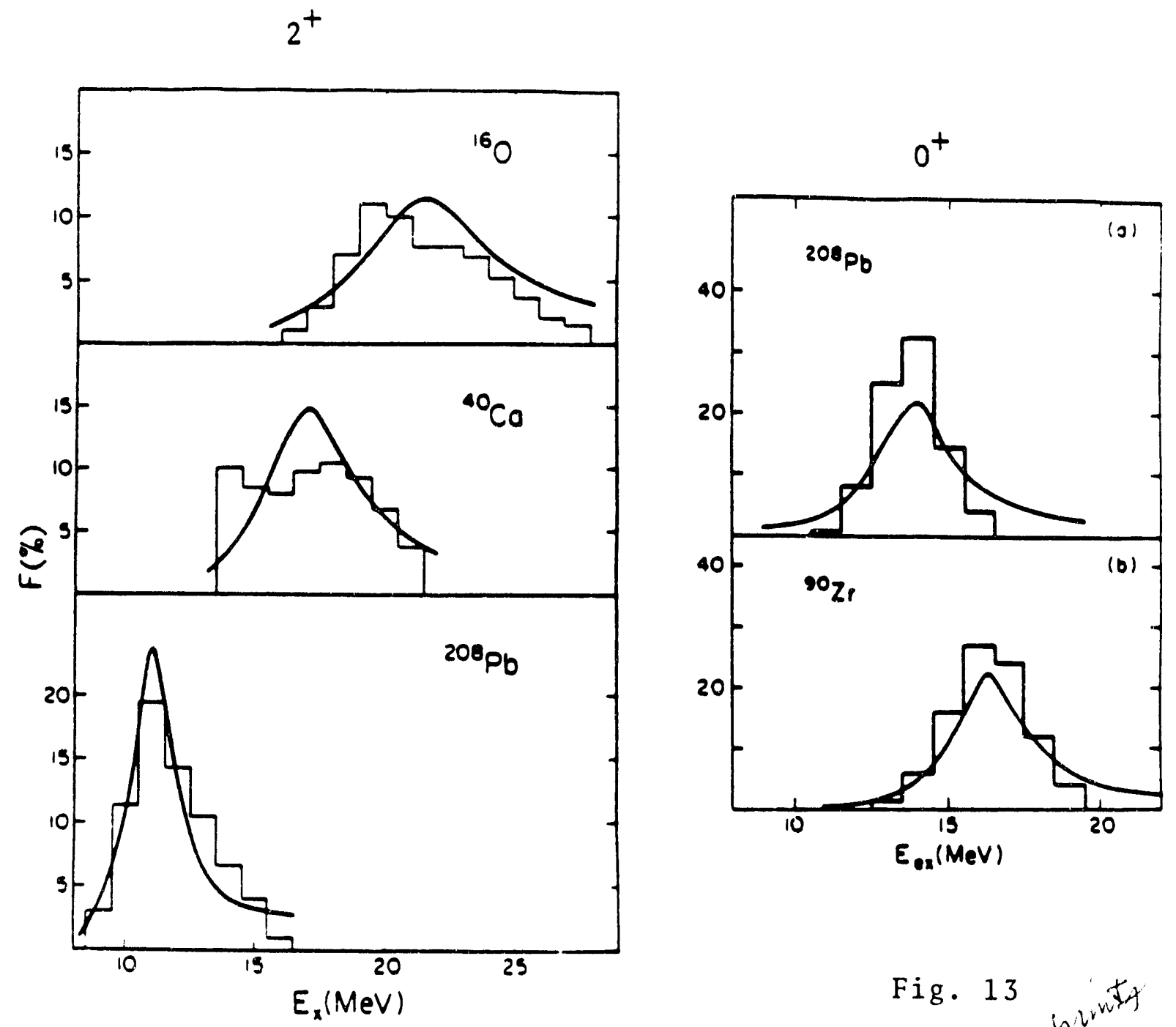

Fig. 12

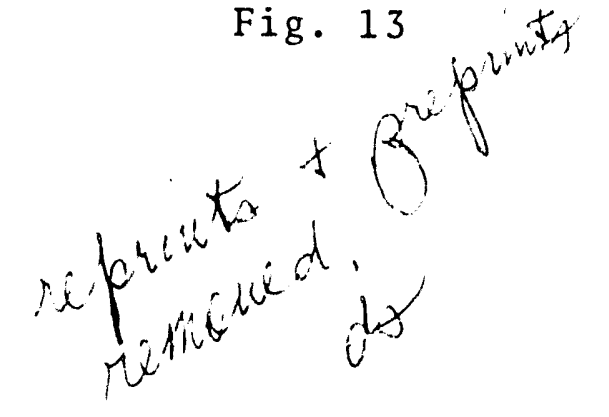




\section{List of Papers (October 1991 - September 1992)}

"Random-Phase Approximation Calculations of the Nuclear Response in the Continuum Using a Finite-Range Interaction", D. Knobles and T. Udagawa, Nucl. Phys. A533, 189 (1991).

"Dynamical Fusion and Direct Reaction Polarization Potentials for Heavy-Ion Scattering and Fusion at Sub-Coulomb-Barrier Energies", B. 'T. Kim and T. Udagawa, Phys. Lett. B 273, 37 (1991).

"RPA Calculations of Nuclear Response in the Continuum for the Hadronic Inelastic Scattering", B. T. Kim and T. Udagawa, Chinese Journal of Physics, 29, 431 (1991).

"Delta Excitations in Nuclei and Its Decay", T. Udagawa, F. Osterfeld, P. Oltmanns, and S. W. Hong, Proceedings of 6-th International Conference on Nuclear Reaction Mechanisms, ed. by E. Gadioli (Universita Delistudi di Milano, Milano, 1991), p.632.

"Systematics of Sub- and Near-Barrier Fusion Cross Sections and the Threshold Anomaly", T. Udagawa, M. Naito, and B. T. Kim, Phys. Rev. (45, 876 (1992).

" $\Delta$-Excitations in Nuclei", F. Osterfeld, B. Körfgen, P. Oltmanns, and T. Udagawa , Proceedings of the Workshop on Meson Production, Interaction and Decay, ed. by A. Magiera, W. Oelert, and E. (irosse (World Scientific, 1991), p.116.

"I Excitations in Nuclei", T. Udagawa, P. Oltmanns, and F. Osterfeld, Proceedings of the International ('onference on Spin and Isospin in Nuclear Interactions, ed. by S. W. Wissink, C. D. Cioodman, and (i. E. Walker (Plenum Press, New York, 1991), p. 69.

"A Unified Approach for Low-Energy Nuclear Reactions", B. T. Kim and T. Udagawa, Proceedings of International Conference on Towards a Unified Picturc of Nuclcar Dynamies, ed. by Y. Abe, S. M. Lee, and F. Sakata, (American Institute of Physics, New York, 1991), p.133.

"Breakup-Fusion Analyses of the ${ }^{40}\left(\mathrm{Ca}\left({ }^{6} \mathrm{Li}, \mathrm{d}\right){ }^{44} \mathrm{Ti}\right.$ Reactions and $\alpha$-('luster Structure in ${ }^{44} \mathrm{Ti} "$, Chong-Yeal Kim and T. Udagawa, Phys. Rev. (' 46, 5i32 (1992). 


\section{PERSONNEL}

\section{Faculty}

Dr. Takeshi Udagawa, Professor

\section{Post-Doctoral Appointments}

Dr. D. Knobles (- February, 1992)

Dr. C. Y. Kim (January, $1992-$ )

\section{Pre-Doctoral Appointments and Graduate Students}

K.-P. Cheng (Fully supported by Chinese goverment, - August, 1990)

A. Gomez-Chamacho (- August, 1991)

M. Naito (- August, 1992)

C.-Y. Kim (- December, 1991)

J. Young (December, 1991 -)

\section{Visitors}

Professor S. W. Hong (July - August, 1991 and July, 1992); (Department of Physics, Sung Kyung Kwan University, Seoul, Korea)

Professor B. T. Kim (December, 1989 - December 1990 and July - August, 1991);

(Department of Physics, Sung Kyung Kwan University, Seoul, Korea)

Dr. R. C. Mastroleo (December, 1989 - February, 1990 and September, 1991 August, 1992);(Instituto de Estudos Avancados, Sao Jose dos Campos, Brazil) 

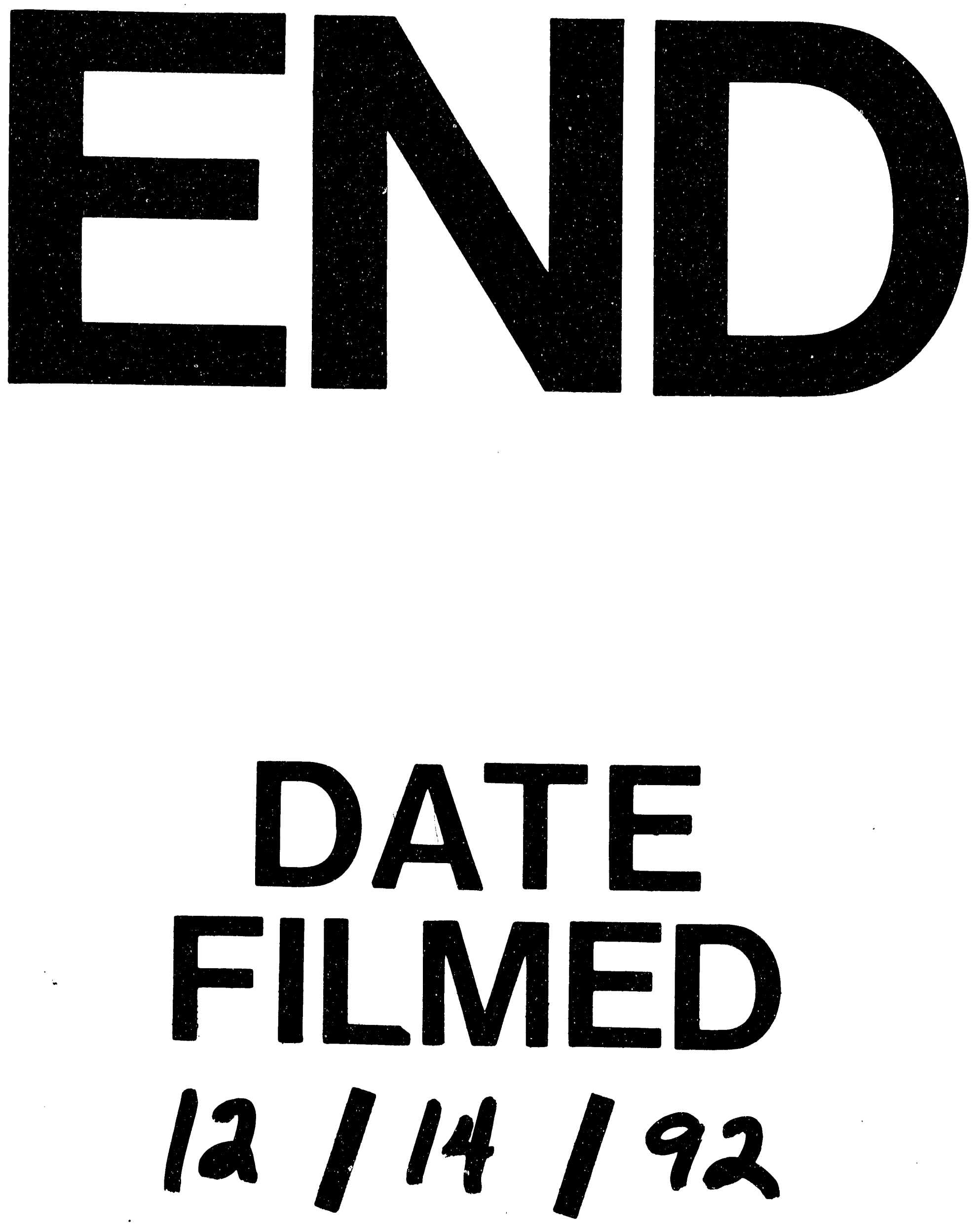
